\title{
Induction of a Regular Nuclear Lattice by Overexpression of NuMA
}

\author{
Catherine Gueth-Hallonet, ${ }^{1}$ J ian Wang, J ens Harborth, Klaus Weber, and Mary Osborn ${ }^{2}$ \\ Department of Biochemistry, Max Planck Institute for Biophysical Chemistry, Am Fassberg 11, 37077 Goettingen, Germany
}

\begin{abstract}
Transient overexpression of nuclear mitotic apparatus protein (NuMA) in HeLa cells results in ordered lattices which can fill the nucleus and which are stable to detergent extraction. Electron microscopy reveals a quasi-hexagonal organization with an average spacing between the vertices of $\sim 170 \mathrm{~nm}$ and short 6-nmdiameter rods connecting the vertices. Overexpression of a NuMA construct with an in-frame addition in the coiled-coil domain shows hexagons with the spacing increased by $42 \%$ while constructs with deletions in the coiled-coil domain yield hexagons with the spacing decreased by 40 and 19\%. NuMA constructs truncated at residue $\mathbf{2 0 0 5}$ or $\mathbf{2 0 3 0}$ in the tail domain cause a drastic reorganization of nuclear components with relocation of the DNA, histone $\mathrm{HI}$, and nucleoli to the nuclear rim. A construct lacking the head and much of the coiled-coil region also affects nuclear organization. In contrast, NuMA constructs truncated at residue 1950 or 1935 which lack the nuclear localization signal display normal nuclear structure but form cytoplasmic aggregates which also display hexagonal organization. I mmunoelectron microscopy confirms that the nuclear lattices are built from NuMA. We discuss the importance of the different domains of NuMA for building the ordered in vivo lattices and whether NuMA could play a structural role in the architecture of the normal interphase nucleus. $\odot 1998$ Academic Press

Key Words: nuclear matrix; NuMA; mitotic spindle; coiled-coil proteins.
\end{abstract}

\section{INTRODUCTION}

Eukaryotic nuclei are responsible for complex and vital functions in interphase such as DNA replication and RNA transcription and processing. The nuclear structure ensures the crucial temporal and spatial coordination of these processes, through the specific attachment of highly condensed chromatin organized into discrete loops to a nonchromatin scaffold referred to as the nuclear matrix or nucleoskeleton. To understand the molecular

\footnotetext{
${ }^{1}$ Current address: École Normale Supérieure, 46, Rue d'UIm, 75005 Paris, France.

2 To whom correspondence and reprint requests should be addressed. Fax: +49551 201 1578. E-mail: mosborn@gwdg.de.
}

composition of the scaffold as well as how these interactions are achieved and regulated during interphase remains a major challenge in cell biology.

The nuclear matrix was originally defined as the insoluble and salt-resistant proteinaceous nuclear structure isolated from interphase cells after digestion with nucleases. Various protocols have been employed to try to observe the nuclear matrix in situ. Some are modifications of the original Berezney and Coffey technique $[1,2]$ while others have removed chromatin by extracting with lithium diiodosalicyate [3] or by using restriction endonucleases and electrophoresis to remove unattached fragments [4]. In some protocols RNA is removed by RNase digestion [5] while in others it is preserved by the use of RNA inhibitors [6, 7]. Such preparative techniques remove almost all the chromatin and most nuclear proteins, as judged by the absence of histone proteins and the predominance of lamins in the final preparations. However, the efficiency of these preparations in retaining the overall features of the intact nuclear matrix ultrastructure, as assessed by electron microscopy, is not clear for two reasons. First, the matrix is hidden by the chromatin in intact cells and thus the relevance of the three-dimensional nuclear matrix composed of long ribonucleoproteinaceous branched filaments of $\approx 10 \mathrm{~nm}$ in diameter observed in some studies is still controversial $[4,7,8]$. Second, the protein composition of the core filaments remains to be determined. Indeed, although several nuclear matrix proteins have been described [9-11], thus far only lamins have been indicated to be major structural components of the core filaments by immunoelectron microscopy [12]. Nor have any of the putative nuclear matrix proteins been shown to polymerize into filaments in vitro.

NuMA (nuclear mitotic apparatus protein) ${ }^{3}$ has some of the characteristics that might be expected for a nuclear matrix protein. This protein is characterized by a cell cycle-dependent localization [13-15]: it locates to the nucleus in interphase, associates with the centrosomes when the nucleus disassembles in prophase, and is found at the spindle poles during later stages of mitosis. NuMA may play a structural role in the archi-

\footnotetext{
${ }^{3}$ Abbreviations used: FL, full length; NLS, nuclear localization signal; NuMA, nuclear mitotic apparatus protein.
} 
tecture of the interphase nucleus and in addition is involved in the formation and maintenance of the mitotic spindle and asters [14, 16-20]. A structural role for NuMA in the architecture of the interphase nucleus is supported by the following results: (i) NuMA is present in the interphase nuclei of almost all cells thus far tested, irrespective of the differentiation state [21, 22; for exceptions see 23]. (ii) NuMA is found throughout the nucleoplasm, with the exception of the nucleoli, and is a component of the nuclear matrix [13, 14]. Although immunogold labeling with a single NuMA antibody suggested that NuMA might be found associated with core filaments of the nuclear matrix [24], this result could not be confirmed in other studies (Nickerson and Penman, unpublished observations cited in [25], see also [26]). (iii) Microinjection of NuMA antibodies, or expression of truncated forms of NuMA, leads to assembly of micronuclei in daughter cells [14, $27,28]$. (iv) NuMA binds specifically in vitro to DNA sequences which are thought to fasten the chromatin to the nuclear matrix [29]. (v) NuMA has a long central coiled-coil region [15, 30, 31] which can also be visualized in the electron microscope [32]. Thus by analogy with other coiled-coil proteins, such as myosin and intermediate filaments, NuMA might be expected to be a filament-forming protein. Indeed cells transfected with a NuMA construct lacking the nuclear localization signal (NLS) accumulate NuMA in cytoplasmic masses with a defined ultrastructure [33]. In addition NuMA assembles into higher order structures when a mitotic extract is dephosphorylated [34]. In vitro studies with recombinant NuMA have revealed dimers but no linear filaments [32].

Thus far N uMA has not been shown to form morphologically recognizable structures within the nucleus. Here we show using immunofluorescence, electron microscopy, and immunoelectron microscopy on detergent extracted cells that overexpression of recombinant full-length (FL) NuMA and of different NuMA constructs results in a drastic modification of the normal nuclear ultrastructural organization. Nuclei of cells transfected with FL NuMA or with NuMA constructs with an in-phase addition or deletion in the coiled-coil region show ordered lattices which fill the nuclei. These lattices have a quasi-hexagonal organization, and the size of the hexagons depends on the length of the coiled-coil in the NuMA construct. We have also analyzed the importance of the different domains of NuMA for building the lattice by using constructs truncated in the tail domain or with a deletion in the head and part of the coiled-coil domain.

\section{MATERIALS AND METHODS}

Construction of the mutant NuMA cDNAs. Figure 1 shows the different NuMA constructs used in this study. Derivation of the
C-terminal truncated NuMA cDNAs ( $\triangle$ NuMA) has been described [28]. To construct NuMA dHCC, a deletion covering amino acid residues 7 to 1502 corresponding to nucleotides 176-4663 was obtained by digesting the pCMV 5 vector containing FL NuMA CDNA with Smal and the digested vector was religated with the digest product corresponding to the C-terminal end. For NuMA dCC1, a deletion covering amino acid residues 870 to 1462 was obtained by excising the NuMA fragment comprising nucleotides 2760-4538 obtained after NgoMl digestion of the FL construct in pCMV5. To obtain NuMA dCC2, a deletion covering amino acid residues 631 to 1502 was obtained by ligating the NuMA fragment comprising nucleotides 176-2047 obtained after Smal digestion into the Smal site of the dHCC construct described above. To obtain the extralong construct CCXL, amino acid residues 496 to 1706 were added by ligating a fragment comprising nucleotides $1644-5276$ obtained after Dral and Hincl digestion into the Hincl site of the FL NUMA construct. To obtain the FL NuMA-c-myc construct, a c-myc tag was added in frame at the $3^{\prime}$ end of FL NuMA CDNA using PCR. The antisense oligo contained a stop codon followed by the sequence coding for the myc epitope EQKLISEEDL followed by the NUMA nucleotide sequence 6501 to 6481 . Sequencing was performed to check the ligated regions.

Cell cultureand transfection. Human HeLa SS6 cells were grown in DME medium plus $10 \%$ fetal calf serum in a humidified incubator at $37^{\circ} \mathrm{C}$ with $5 \% \mathrm{CO}_{2}$. HeLa cells were diluted $24 \mathrm{~h}$ prior to transfection and at the time of transfection, were about $50 \%$ confluent. They were transiently transfected using the modified calcium phosphate precipitation technique [35]. Briefly, $14 \mu \mathrm{g}$ of plasmid DNA was washed with ethanol, lyophilized, and dissolved in $300 \mu \mathrm{l}$ of a $250 \mathrm{mM}$ calcium chloride solution. Three hundred microliters of 50 $\mathrm{mM}$ BES-buffered saline $(50 \mathrm{mM}$ BES, $250 \mathrm{mM} \mathrm{NaCl}, 1.5 \mathrm{mM}$ $\mathrm{Na}_{2} \mathrm{HPO}_{4}, \mathrm{pH}$ 6.96) was then added. After 10 min at room temperature, this solution was added dropwise to the cells in a $60-\mathrm{mm}$ diameter petri dish containing $3 \mathrm{ml}$ of medium. After 12 to $18 \mathrm{~h}$ at $35^{\circ} \mathrm{C}$ with $3 \% \mathrm{CO}_{2}$, cells were washed with prewarmed PBS. Five milliliters of prewarmed medium was then added and the cells were returned to $37^{\circ} \mathrm{C}$ with $5 \% \mathrm{CO}_{2}$. When specified $2 \mu \mathrm{g} / \mathrm{ml}$ colcemid (Sigma $\mathrm{GmbH}$, Deisenhofen) was added for $2 \mathrm{~h}$ to cells $40 \mathrm{~h}$ after transfection.

SDS gel electrophoresis and immunoblotting. Whole-cell extracts were harvested in sample buffer [28] separated on $4 \%$ (for all constructs but $\mathrm{dHCC}$ ) or $7 \%$ (for the $\mathrm{dHCC}$ construct) SDS polyacrylamide gels and transferred el ectrophoretically to nitrocellulose. Immunostaining with undiluted hybridoma supernatant from the SPN7 clone and rabbit anti-mouse peroxidase-labeled antibodies (Dako Hamburg) (1:5000 dilution) was carried out using the ECL technique (Amersham, Braunschweig).

In situ extraction. HeLa SS6 cells were rinsed in a microtubule stabilizing buffer (4 M glycerol, $100 \mathrm{mM}$ Pipes, pH 6.8, 1 mM EGTA, $5 \mathrm{mM} \mathrm{MgCl}$ ) for 30-60 s at room temperature $[33,36]$. They were then extracted for 2-3.5 min in buffer containing $0.5 \%$ Triton X-100 at room temperature and rinsed in buffer without Triton for $30 \mathrm{~s}$ before fixation either with methanol for immunofluorescence microscopy or with glutaraldehyde for electron microscopy (see below).

Immunofluorescence microscopy. Cells were grown on $12-\mathrm{mm}-$ diameter glass coverslips and fixed in methanol for $6 \mathrm{~min}$ at $-10^{\circ} \mathrm{C}$. I mmunofluorescence was carried out by standard procedures. Transfected cells expressing FL or one of the constructs could be identified directly using an appropriate NuMA antibody (see below). For the lamin $\mathrm{A} / \mathrm{C}$ and histone $\mathrm{H} \mathrm{l}$ staining of cells expressing $\mathrm{FL}, \mathrm{dHCC}$ or $\Delta 2005$, transfected cells were identified by their altered staining patterns. To detect cell death in situ, cells were labeled with dUTPFITC for $1 \mathrm{~h}$ with the TUNEL-FITC kit (Boehringer Mannheim $\mathrm{GmbH}$, Mannheim) prior to the NuMA staining. At the end of the procedure, cells were stained for $4 \mathrm{~min}$ at room temperature with Hoechst 33342 (1:200 dilution in PBS from a $1 \mathrm{mM}$ stock solution in water) and embedded in Mowiol 4.88 (Hoechst AG, Frankfurt). 
a
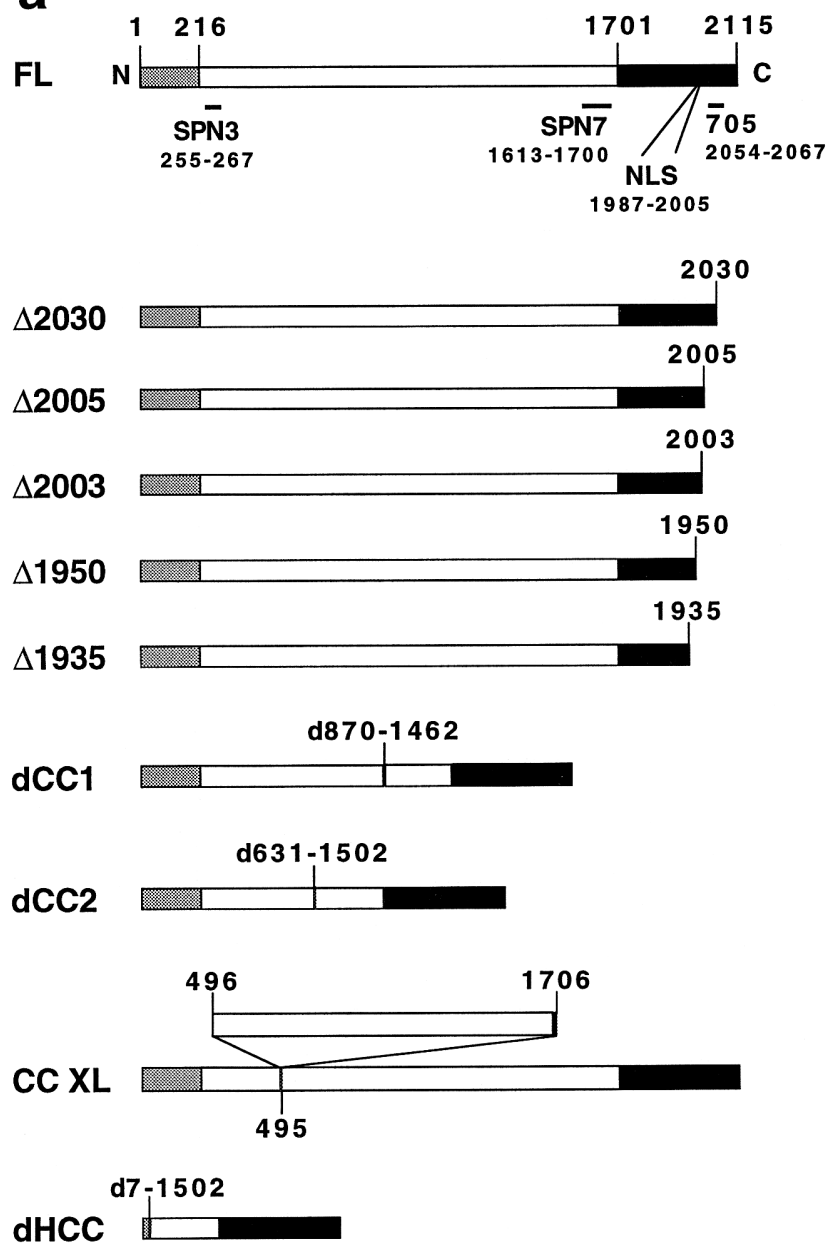

b

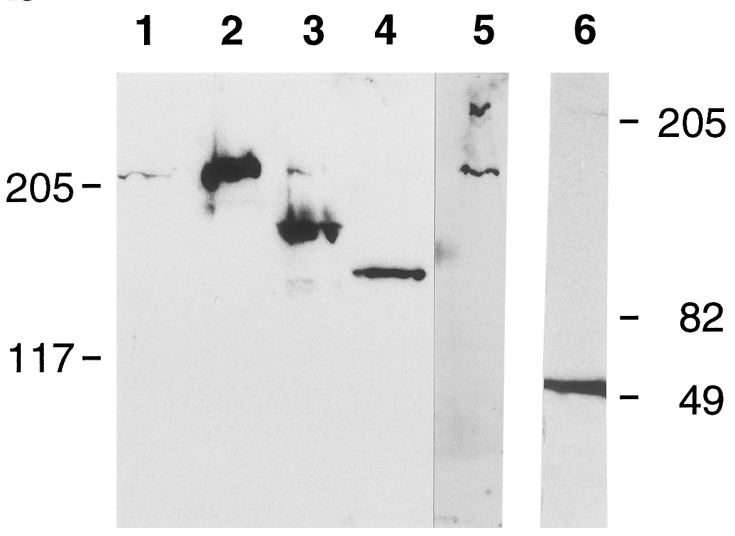

FIG. 1. (a) Schematic representation of the secondary structure of recombinant full-length (FL) or mutant NuMA polypeptides. $(\square)$ Head, ( $\square$ ) coiled-coil rod domain, (घ) tail. The amino acid numbering system is from Yang and Snyder [17] and the first residues of the head, coiled-coil, and tail domains are shown. The positions of the nuclear localization signal (NLS) and the epitopes of the antibodies
Antibodies. Monoclonal NuMA SPN3 and SPN7 antibodies [14, 32], the monoclonal histone $\mathrm{H} 1$ antibody (clone $\mathrm{AE}-4$, Cal biochem), the monoclonal 20C6 tubulin antibody [37], and the monoclonal 636 lamin A/C-specific antibody [38] were used as undiluted hybridoma supernatants. Monoclonal c-myc antibody (clone 9E 10, Santa Cruz Biotechnology, Heidelberg) was used diluted 1 to 50. The rabbit NuMA 705 antibody was raised by injecting a synthetic peptide covering residues 2176 to 2189 in the frog NuMA tail coupled to oval bumin. This sequence [20] differs by a single amino acid change ( $\mathrm{K}$ instead of R) at position 2187 from the corresponding human sequence which covers residues 2054 to 2067 [30]. The antibody was affinity-purified on the peptide coupled to EAH Sepharose 4B with sulfo-MBS by a cysteine residue added at the amino-terminus and was used at a concentration of $20 \mu \mathrm{g} / \mathrm{ml}$. Human autoimmune NuMA serum 2422 (a kind gift from Professor Karsten Conrad, University of Dresden) was diluted 1 to 1000.

Transmission electron microscopy. Cells on 12-mm glass coverslips were extracted as above and then fixed in glutaraldehyde ( $2 \%$ in $0.1 \mathrm{M}$ cacodylate, $\mathrm{pH} 7.2,0.1 \mathrm{M}$ sucrose) for $45 \mathrm{~min}$ at room temperature. They were fixed with osmium tetroxide ( $2 \%$ in the same buffer) for $30 \mathrm{~min}$ on ice. They were rinsed several times with $0.1 \mathrm{M}$ cacodylate, $\mathrm{pH} 7.2$, containing $0.1 \mathrm{M}$ sucrose. Some cultures were stained en bloc with uranyl acetate ( $1 \%$ in water) for $60 \mathrm{~min}$ on ice. The cells were dehydrated in a graded series of ethanol and propylene oxide and flat-embedded in Epon. After polymerization of the Epon the coverslips were removed by etching in $48 \%$ hydrofluoric acid for $20 \mathrm{~min}$ at room temperature. They were rinsed three times in distilled water and dried at $60^{\circ} \mathrm{C}$ for $2 \mathrm{~h}$. The flat-embedded cells were sectioned serially parallel to the substratum. Sections were stained with uranyl acetate and lead citrate and examined in a Phillips CM 12 transmission electron microscope.

Measurements. Hexagons with clearly distinguishable electrondense particles at each of the six vertices were chosen at random on the $17,000 \times$ micrographs and the 7 vertices (6 peripheral and 1 central) were marked. For each hexagon, 6 central to peripheral distances and 6 peripheral to peripheral distances were measured. The average of these 12 values was calculated and used to characterize the hexagon size. A minimum of 7 hexagons chosen on three different transfected cells were analyzed in this way for cells transfected with each of the NuMA constructs. Round particles were selected for measurement of the particle diameter. The particle diameter was measured by superimposing computer-drawn circles on a transparent foil differing by $0.1 \mathrm{~mm}$ in diameter on a particle until a circle was found in which the particle filled the field with no visible white space.

I mmunogold labeling. For the nanogold preembedding immunoelectron microscopy HeLa SS6 cells transfected with the FL NuMA construct and untransfected cells were subjected to the in situ extraction procedure given above. They were then fixed with either $2 \%$ paraformaldehyde or $0.1 \%$ glutaraldehyde for $10 \mathrm{~min}$ before being subjected to the procedure described in [39]. For these experiments either IgGs purified from SPN3 ascites fluid at a dilution of 20-40 $\mu \mathrm{g} / \mathrm{ml}$ or SPN3 supernatant was used. Nanogold-labeled goat antimouse I gGs (Nanoprobes I nc., Stony Brook, NY) at a dilution of 1:10

SPN3, SPN7, and 705 are given under the FL sequence. (b) I mmunoblot detection of FL and mutant human NuMA $36 \mathrm{~h}$ after transfection of HeLa cells. The NuMA SPN 7 monoclonal antibody detected both the endogenous and the recombinant human proteins. (Lane 1) Mock transfected; (lane 2) FL transfected; (lane 3) dCC1 transfected; (lane 4) dCC2 transfected; (lane 5) CCXL transfected; (lane 6) dHCC transfected. The transfection rates calculated from parallel cultures stained in immunofluorescence microscopy were 19,20,11, 3, and $20 \%$ for lanes 2-6, respectively. Molecular weight standards are shown on the left for lanes 1 to 5 and on the right for lane 6 . 
to 1:30 was used as the second antibody. Incubation with the first antibody was carried out for $1.5 \mathrm{~h}$ at room temperature and for the second antibody was carried out for $13 \mathrm{~h}$ at $4^{\circ} \mathrm{C}$ followed in some experiments by $2 \mathrm{~h}$ at room temperature. Controls were carried out by omitting the first antibody. Samples were postfixed in $2.5 \%$ glutaraldehyde and developed with the HQ silver development kit (Nanoprobes Inc) for 5- $6 \mathrm{~min}$ at room temperature. The samples were stained with $0.2 \%$ osmium tetroxide for $30 \mathrm{~min}$ at $4^{\circ} \mathrm{C}$ and after dehydration were embedded in Epon resin. Grids were stained with uranyl acetate.

\section{RESULTS}

HeLa cells were transfected with each of the NuMA constructs shown in Fig. 1a with mock transfected Hela cells acting as a control. Then, 36-42 h after transfection cells were stained with NuMA antibodies to assay the frequency of transfection and to determine the NuMA staining patterns (cf. patterns shown in Fig. 2 for the $F L$ construct). Three different antibodies were used: (i) the monoclonal antibody SPN 3 which recognizes an epitope at the beginning of the coiled-coil region (residues 255-267); (ii) the monoclonal antibody SPN 7, which recognizes an epitope at the end of the coiled-coil region (residues 1613-1700); and (iii) a new NuMA rabbit antibody, 705, which recognizes an epitope in the tail domain (residues 2054 to 2067) (Fig. $1 \mathrm{a}$ and [32]). Cells were also stained with the lamin A/C 636 antibody [38] to determine the lamin distribution and with a histone $\mathrm{HI}$ antibody to determine histone distribution. Parallel cultures were extracted with a microtubule stabilizing buffer containing $0.5 \%$ Triton $\mathrm{X}-100$. They were then examined using immunocytochemistry with antibodies to NuMA in the light microscope or alternatively were fixed in glutaral dehyde and embedded as monolayers for electron microscopy. After being sectioned, control cultures and cultures transfected with each construct were screened at a magnification of $3000 \times$ to look for changes in nuclear structure.

\section{Nuclear Structure in Normal Untransfected Cells}

In interphase HeLa cells NuMA was found by immunofluorescence microscopy throughout the nucleoplasm with the exception of the nucleolus, while in mitotic cells NuMA stained the spindle poles. The staining of interphase nuclei with SPN3 (Fig. 3a) and SPN 7 antibodies (data not shown) was usually homogenous although occasionally a dotted impression was obtained. In contrast, the NuMA antibody 705 clearly stained the nucleoplasm in a dotted fashion (Fig. 3c). All interphase nuclei had approximately the same intensity when stained with a given antibody. The patterns were not changed by extraction with detergent with the exception that the dotted staining became slightly more prominent (compare Fig. 3f with Fig. 3a and Fig. $3 g$ with Fig. 3c). The retention of NuMA in
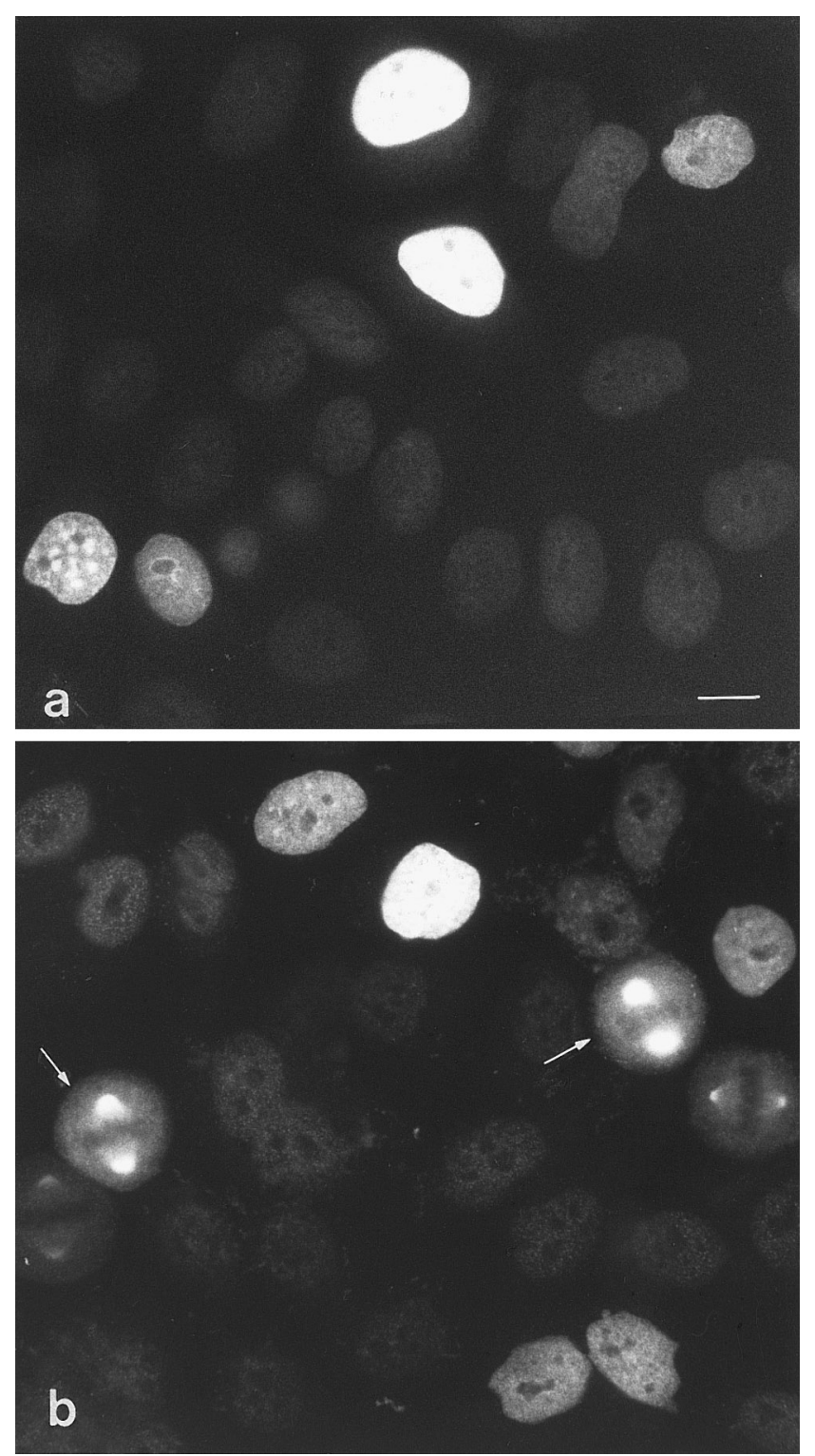

FIG. 2. Overview of immunofluorescence staining of transfected cells $36 \mathrm{~h}$ after transfection. (a) NuMA SPN 3 antibody; (b) NuMA 705 antibody. Transfected cells express different amounts of NuMA. (a) Five transfected cells and (b) seven transfected cells with many untransfected cells present in both panels. Note in (b) that the two transfected metaphase cells (arrows) are much more strongly stained than the two untransfected metaphase cells present in the same panel. Bar $(a, b) 10 \mu \mathrm{m}$.

Triton-treated cells is in agreement with our previous immunoblotting data showing that NuMA is retained in the pellet fraction and is not present in the soluble fraction after Triton X-100 extraction (Fig. 1b in [14]). Lamin $\mathrm{A} / \mathrm{C}$ and histone $\mathrm{H} 1$ staining patterns in unextracted cells are shown in Figs. $3 d$ and $3 e$, respectively.

When Hela cells extracted with detergent buffer were examined in the electron microscope, nucleoli, patches of chromatin, and residual proteinaceous 


\section{NuMA SPN3}
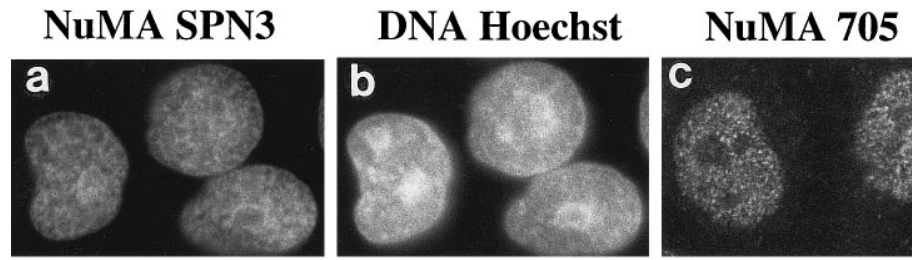

Lami
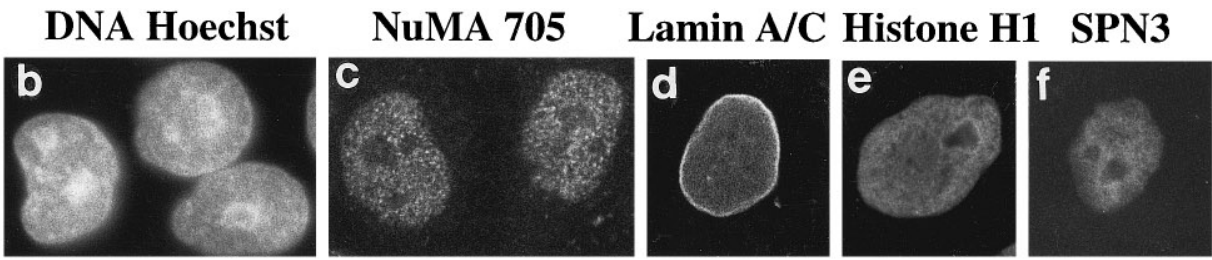

705

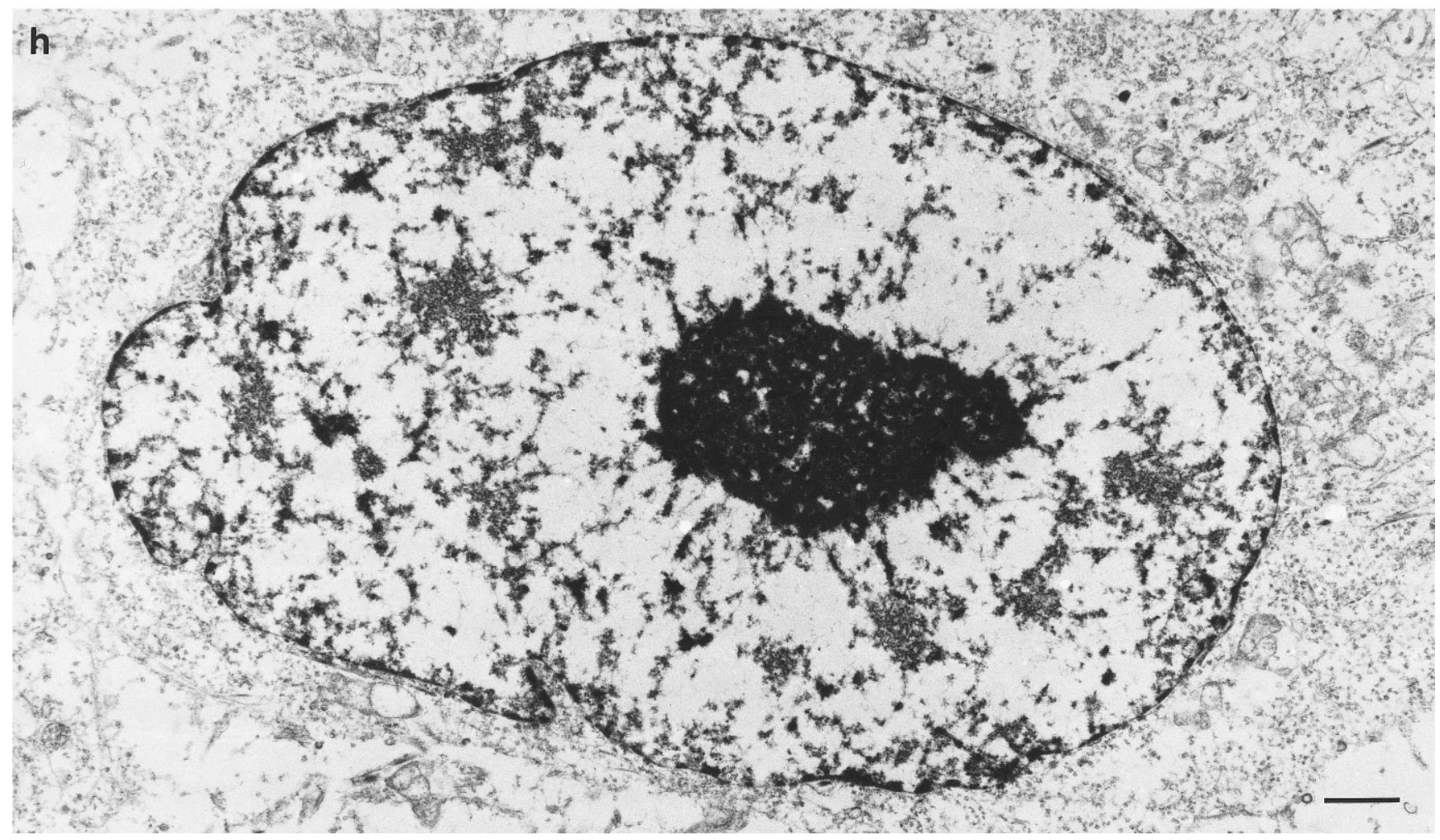

FIG. 3. Immunofluorescence staining (a-g) and electron microscopy after detergent extraction (h) of HeLa cells $42 \mathrm{~h}$ after mock transfection. (a, b) Cells double- stained with NuMA SPN3 antibody (a) and Hoechst dye (b). (c) NuMA 705 antibody staining. (d) Lamin A/C staining. (e) Histone $\mathrm{H} 1$ staining. (f, g) SPN3 (f) or 705 (g) staining after detergent extraction. Note that the NuMA 705 antibody gives a dotted pattern in the interphase nucleus. Note that no regular substructure is visualized in $(h)$ and that the nucleus seems rather empty. Bar (a-g) $10 \mu \mathrm{m}$; (h) $1 \mu \mathrm{m}$.

structures were retained in the nuclei (Fig. 3h). Many nuclei gave a rather empty appearance.

Overexpression of Recombinant FL NuMA and of FL NuMA-C-myc Proteins Results in Formation of Nuclear Lattices

The percentage of cells transfected with the FL construct as assayed by light microscopy varied from 5 to $19 \%$ depending on the experiment. In the overviews shown in Fig. 2 the transfected cells express different amounts of NuMA as shown by staining with SPN3 (Fig. 2a) or the 705 antibodies (Fig. 2b) $36 \mathrm{~h}$ after transfection. Transfected metaphase cells were much more strongly stained than metaphase cells in normal cultures (Fig. 2b). Little detail could be seen in most interphase nuclei stained with the SPN 3 antibody. The 705 antibody, in contrast, stained interphase nuclei of the FL transfected cells in a dotted fashion (Fig. 2b). There were many more dots and the dots were closer together than in untransfected cells stained with the same antibody (compare Figs. 3c and 4c). In addition, a c-myc antibody recognizing a c-myc tag added at the C-terminal end of F L N UMA in the FL C-myc construct also revealed a dotted immunofluorescent staining (Fig. 4d). Lamin A/C and histone $\mathrm{H} 1$ distribution in the cells overexpressing FL NuMA were similar to those in control cells (compare Fig. 4e with Fig. 3d and Fig. $4 f$ with Fig. 3e). I mmunofluorescence microscopy of transfected cells extracted with the detergent buffer showed that the FL NuMA polypeptides were retained in the nuclei (Fig. 4g) (compare to [14, 24]). Recombinant FL NuMA proteins were also retained in the nuclear matrices prepared using the protocol of [7], which includes detergent, DNase, and salt extraction treatments, 

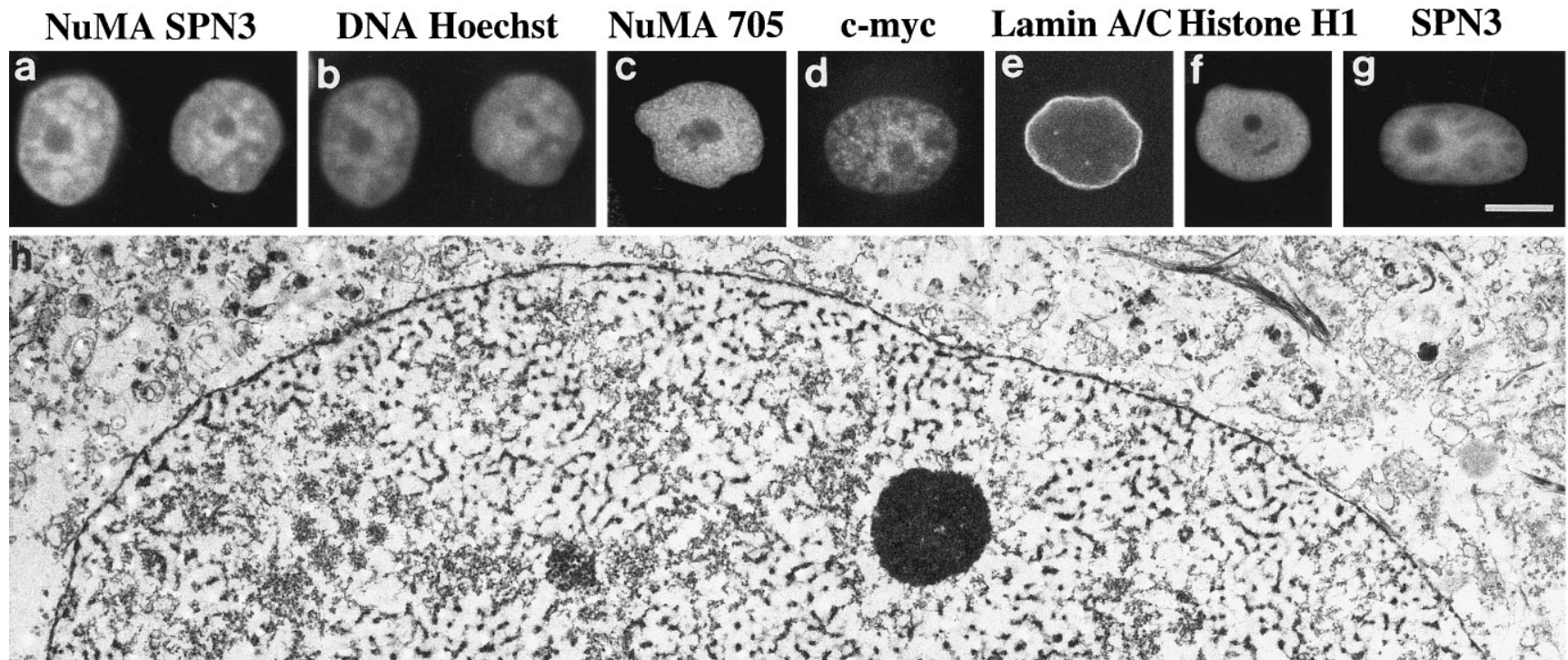

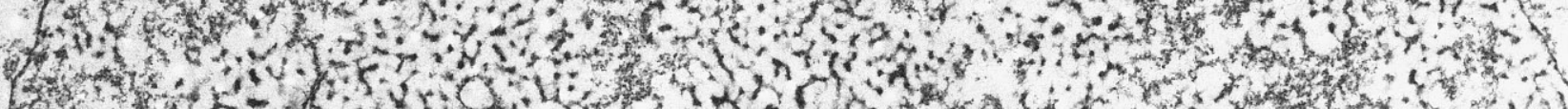

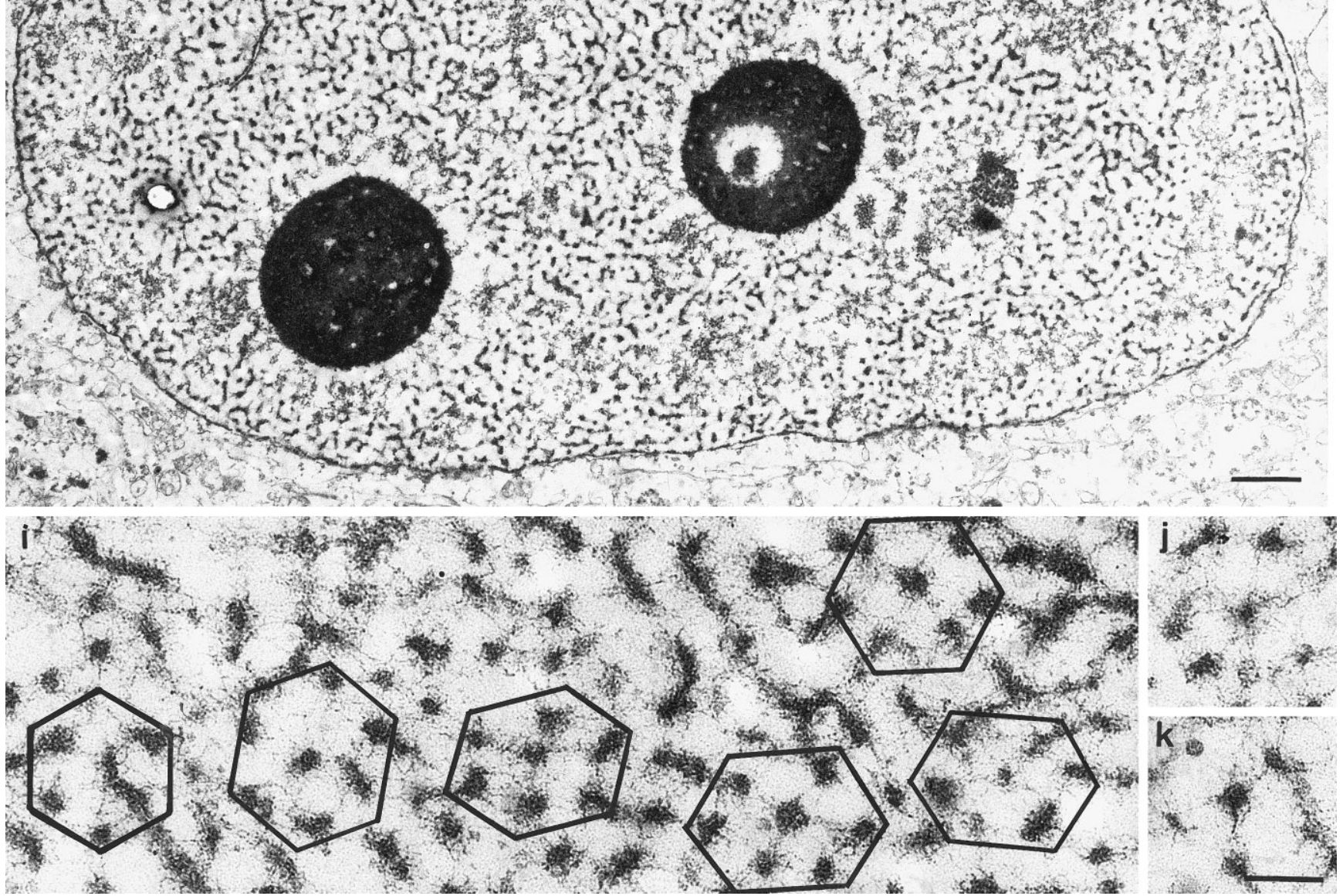

FIG. 4. I mmunofluorescence staining (a-g) and electron microscopy after detergent extraction ( $\mathrm{h}-\mathrm{k}$ ) of HeL a cells $42 \mathrm{~h}$ after transfection with FL NuMA (all except d) or with the FL c-myc CDNA (d). (a. b) Cells double-stained with NuMA SPN3 antibody (a) and Hoechst dye (b). (c) NuMA 705 antibody staining. (d) c-myc antibody staining. (e) Lamin A/C staining. (f) Histone H1 staining. (g) SPN3 staining after detergent extraction. Note the dotted staining seen for NuMA with the 705 and c-myc antibodies. Note the ordered lattice which fills the nucleus in (h). Hexagons are marked in the high-magnification micrographs in (i) and thin rods connecting the hexagon vertices can be seen in (i-k). Hexagons in this and subsequent figures were drawn to illustrate the hexagonal organization. For details of hexagon measurements see Materials and Methods and Table 1. Bar (a)-g) $10 \mu \mathrm{m}$; (h) $1 \mu \mathrm{m}$; (i-k) $200 \mathrm{~nm}$. 


\section{TABLE 1}

Center to Center Distances and Particle Diameters (Mean $\pm \mathrm{SD}$ in $\mathrm{nm}$ ) Measured from the Hexagonal Arrangements of Particles Observed in Electron Micrographs of HeLa Cells Overexpressing FL NuMA or Different NuMA Derivatives

\begin{tabular}{lccc}
\hline $\begin{array}{c}\text { NuMA } \\
\text { construct }\end{array}$ & $\begin{array}{c}\text { Measured center } \\
\text { to center } \\
\text { distance (in nm) }\end{array}$ & $\begin{array}{c}\text { Measured } \\
\text { particle } \\
\text { diameter } \\
\text { (in nm) }^{\text {a }}\end{array}$ & $\begin{array}{c}\text { Expected center } \\
\text { to center } \\
\text { distance (in nm) }\end{array}$ \\
\hline FL & $166 \pm 14$ & $58 \pm 3$ & 279 \\
CCXL & $236 \pm 27$ & $65 \pm 11$ & 466 \\
dCC1 & $135 \pm 20$ & $44 \pm 3$ & 177 \\
dCC2 & $102 \pm 13$ & $41 \pm 3$ & 132 \\
$\Delta 2005$ & $130 \pm 10$ & $26 \pm 3$ & 247 \\
& & & \\
$\Delta 1950^{c}$ & $136 \pm 11$ & $46 \pm 6$ & 267 \\
$\Delta 1935$ & $128 \pm 10$ & $38 \pm 5$ & 259 \\
\hline
\end{tabular}

Note $\Delta 1950$ and $\Delta 1935$ form cytoplasmic aggregates, while the other constructs localize in the nucleus.

a For details of how measurements were made, see Materials and Methods.

${ }^{\mathrm{b}}$ Center to center distance is cal culated using a value of $0.1485 \mathrm{~nm}$ per coiled-coil residue and adding the measured particle diameter.

c After a 2-h treatment with $2 \mu \mathrm{g} / \mathrm{ml}$ colcemid.

again as judged by immunofluorescence experiments (data not shown).

In the electron microscope the vast majority of cell nuclei after extraction with detergent buffer had an appearance similar to that shown for mock transfected cells in Fig. 3h. However, some cells transfected with the FL NuMA construct revealed structures similar to that shown at low magnification in Fig. 4h. Nucleoli and patches of chromatin were clearly distinguishable but in addition a striking network that filled the nucleus could be discerned. At higher magnification this structure appeared to be formed from a three-dimensional network of hexagons with an electron-dense particle at each vertex and an identical particle at the center of the hexagon (Fig. 4i). Particles appeared to be connected by thin filaments for which we measured a diameter of $5.5 \pm 0.5 \mathrm{~nm}$ (Figs. $4 \mathrm{i}, 4 \mathrm{j}$, and $4 \mathrm{k}$ ). $\mathrm{FL}$ NuMA hexagons were characterized by an average interparticle distance of $166 \pm 14 \mathrm{~nm}$ (Table 1 ). The percentage of transfected cells assayed by light microscopy and the percentage of cells showing nuclear lattices were compared directly. In one experiment in immunofluorescence $12.4 \%$ of cells were transfected (1126 cells counted) while in electron microscopy we found the nuclear lattice structure in $1.1 \%$ (or 12/1100) of interphase nuclei. In a second experiment in immunofluorescence $9 \%$ of cells were transfected while in electron microscopy we found the nuclear lattice structures in $1.2 \%$ (5/405). This suggests that the lattice structure is associated only with the cells which, express very large amounts of NuMA.

We stress that hexagonal structures of the type shown in Figs. 4h and $4 \mathrm{i}$ were never found in control cultures. FL transfected cultures could be distinguished from control cultures by the presence of such structures as viewed by a neutral observer unaware of the status of the cultures that were being examined.

\section{NuMA Recombinant Proteins with an In-Phase Addition or Deletion in the Coiled-Coil Region Change the Spacing of the Lattice}

The FL NuMA molecule has a long central coiled-coil region, which by analogy to other coiled-coil molecules may be used as a spacer. It seemed therefore reasonableto see what would happen if constructs with longer or shorter coiled-coil regions were examined. Three different constructs were used (Fig. 1a). One construct-NuMA CCXL_had a duplication of residues 496-1706 which increased the length of the putative coiled-coil region by $80 \%$. The other two constructsNuMA dCCl and NuMA dCC2- had deletions in the coiled-coil region of 40 or $60 \%$, respectively (Fig. 1a). The immunoblots on extracts of cells expressing these new constructs shown in Fig. 1b revealed apparent molecular masses in line with the sizes of 365 (lane 5), 167 (lane 3), and 137 kDa (lane 4) predicted for these constructs from Fig. 1a.

When cells were transfected with the NuMA CCXL, dCC1, or dCC2 constructs, immunofluorescence staining showed NuMA and Hoechst staining patterns similar to those seen in cells transfected with the $F L$ NuMA construct (Figs. 5a, 5b, 5e, and 5f). In the electron microscope, nuclei in which the hexagonal organization could be visualized were found for all three constructs (Figs. 5c, 5d, 5g, and 5h). Measurements showed that the average center to center particle distance was $236 \pm 27 \mathrm{~nm}$ for the CCXL construct (Figs. $5 c$ and 5d; Table 1) and thus considerably larger than that seen with the FL NuMA construct. In contrast, the center to center particle distance was $135 \pm 20 \mathrm{~nm}$ for the dCC1 construct and $102 \pm 13 \mathrm{~nm}$ for the dCC2 construct (Figs. $5 \mathrm{~g}$ and $5 \mathrm{~h}$; Table 1). Thus both constructs gave shorter center to center spacing than that seen with FL NuMA.

The difference in center to center spacing for the hexagons seen with the CCXL and the dCC 2 constructs is particularly striking. Comparison of the low-magnification micrographs in Figs. 4c and $4 \mathrm{~g}$, and of the highmagnification micrographs in Figs. 5d and 5h, emphasizes that the CCXL spacing is almost $2.5 \times$ that of dCC2. 


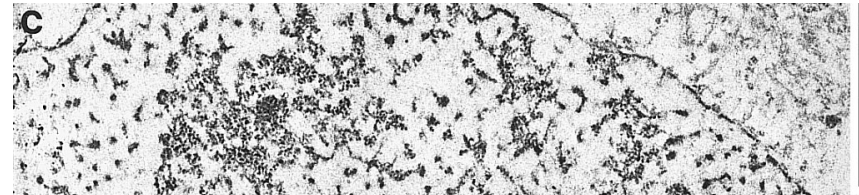

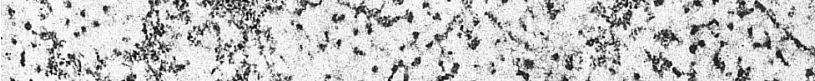

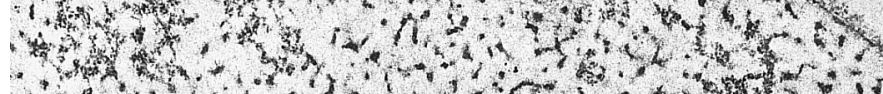
H.

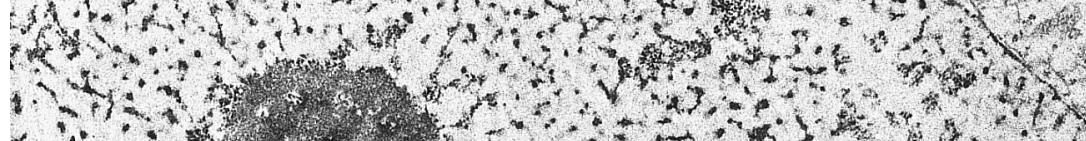

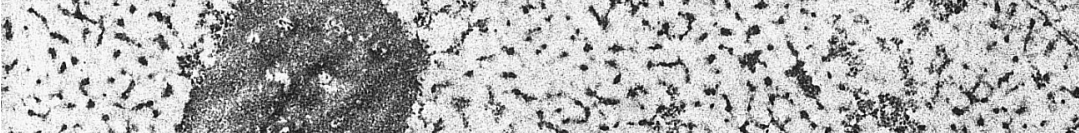

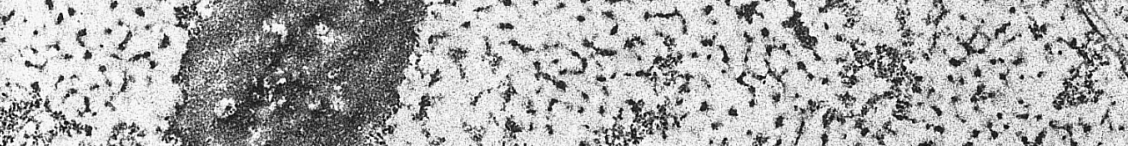

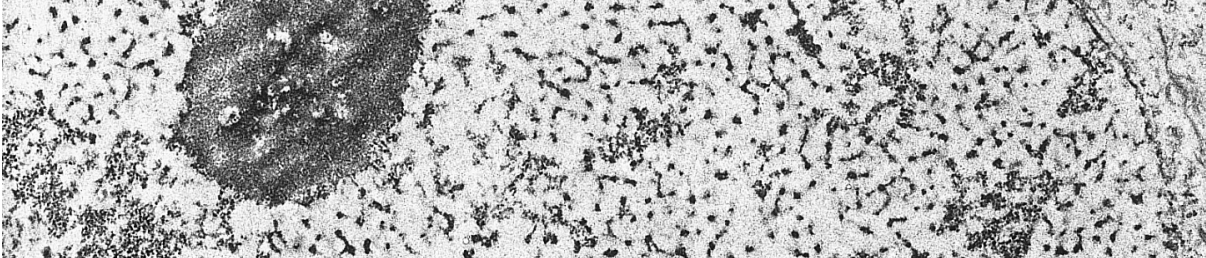

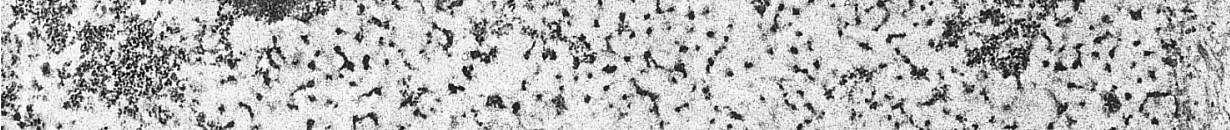

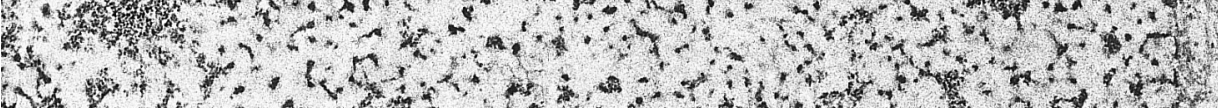

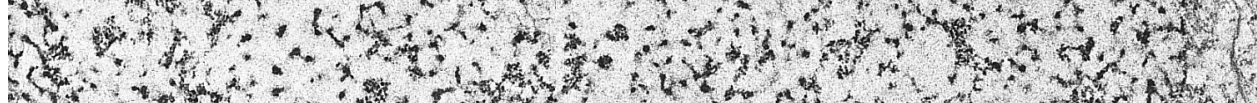

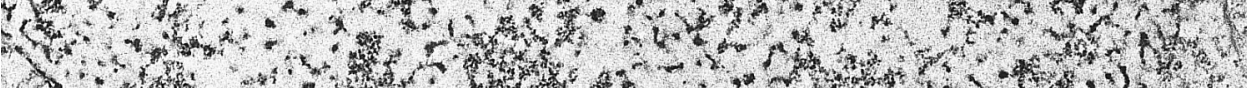

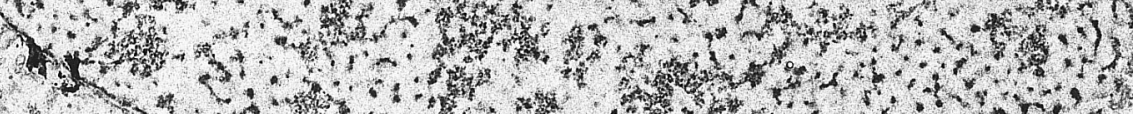

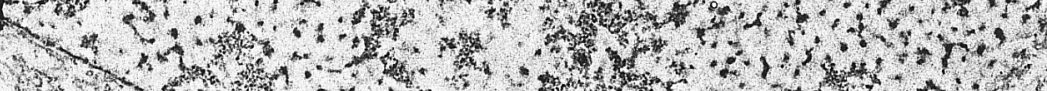

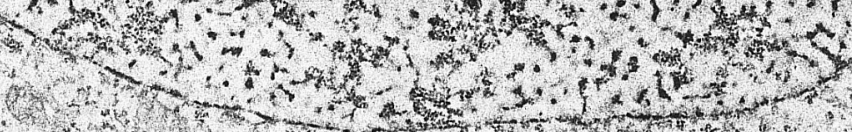

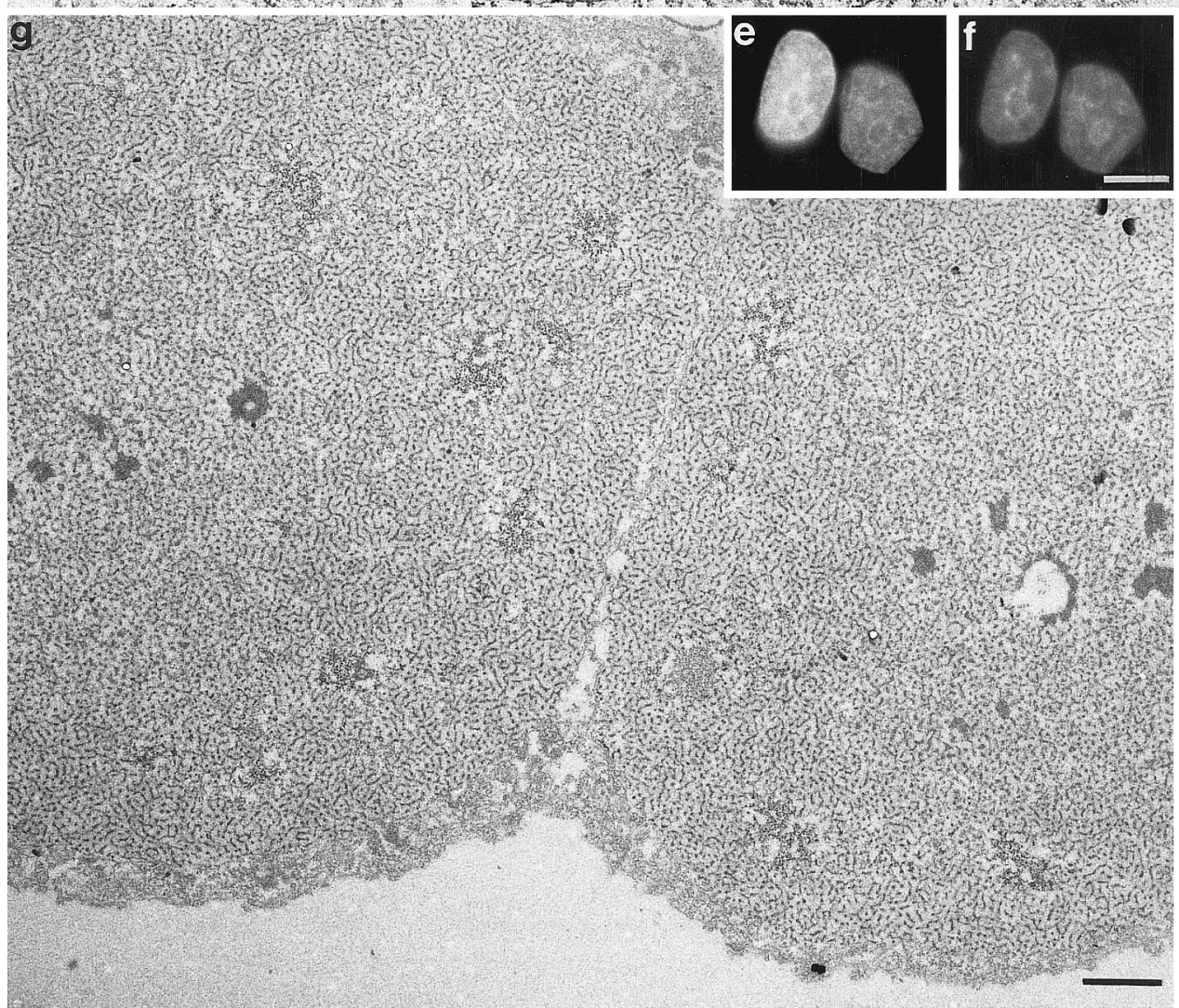

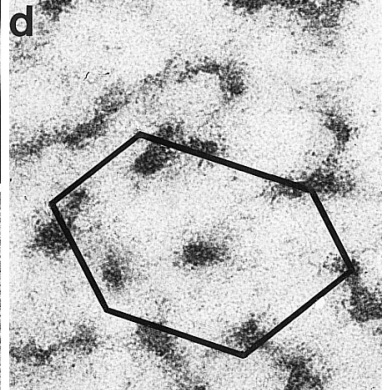
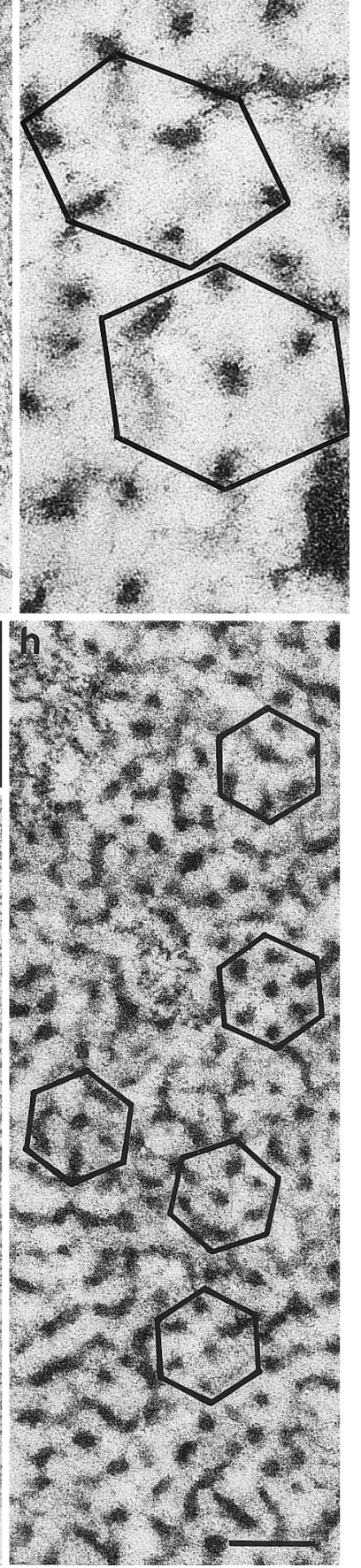

FIG. 5. I mmunofluorescence staining (a, b, e, f) and electron microscopy after detergent extraction (c, d, g, h) of HeLa cells $42 \mathrm{~h}$ after transfection with the CCXL (a-d) or dCC2 (e- h) NuMA CDNAs. In CCXL the length of the coiled-coil was increased by $80 \%$; in dCC2 it was reduced by $60 \%$ relative to FL NuMA. (a, b, e, f) Cells double-stained with NuMA SPN 3 antibody (a, e) and Hoechst dye (b, f). Hexagons are marked in the high-magnification micrographs $(d, h)$. Note that the spacing between the vertices of the hexagons of the CCXL construct (d) is almost $2.5 \times$ the spacing seen with dCC2 construct (h). Bar (a, b, e, f) $10 \mu \mathrm{m} ;(\mathrm{c}, \mathrm{g}) 1 \mu \mathrm{m} ;(\mathrm{d}, \mathrm{h}) 200 \mathrm{~nm}$. 
NuMA Polypeptides with Short C-Terminal

Truncations Have Dramatic Effects on Nuclear Structure

The results thus far obtained suggested that the length of the putative coiled-coil domain was important in determining the spacing of the structures seen in the nuclei that overexpress FL NuMA or NuMA constructs with additions or deletions in the coiled-coil region. All constructs thus far used had intact head and tail domains. We next chose to transfect cells with constructs in which different lengths of the tail region were deleted.

To do this we first induced overexpression of the NuMA $\Delta 2005$ and $\Delta 2030$ constructs which terminate at residues 2005 and 2030, respectively (Fig. 1a). These constructs retain the nuclear location signal and the SPN 3 epitope but have lost the 705 epitope and therefore do not stain with the 705 antibody. In HeLa cells transfected with either the $\Delta 2005$ or the $\Delta 2030$ construct, immunofluorescence microscopy showed one to three large aggregates which fill the nucleus and which stained very strongly with the SPN 3 antibody (F ig. 6a). The NuMA 705 antibody showed staining which was strongest at the outer rim of the aggregates (compare Figs. 6a and 6b with Fig. 6c). Thus endogenous FL NuMA molecules seemed not to participate in the aggregates but rather to abut and envelop them. Interestingly the nucleolus and chromatin as assessed by Hoechst staining were restricted to the outer zone surrounding the aggregates formed from the truncated NuMA proteins (Fig. 6b). Histone $\mathrm{H} 1$ staining was detected only at the nuclear periphery where its distribution was similar to that of the chromatin (Fig. 6e). The lamin A/C distribution appeared normal, however (Fig. 6d). $\Delta 2005$ NuMA polypeptides remained in the nucleus after extraction with detergent buffer (Fig. 6f) or by the method of He et al. [7] (data not shown).

Electron micrographs of cells transfected with the C-terminal truncated $\Delta 2005$ construct are shown in Figs. $6 \mathrm{~g}$ and $6 \mathrm{~h}$. Strikingly and in accordance with the immunofluorescence results, the nucleolus and electron-dense proteinaceous material and less dense chromatin patches were found only at the nuclear periphery. The interior of these nuclei, which correspond to the aggregates seen in the light microscope, shows some material which is not very electron-dense. At higher magnification this material is clearly different from that seen after overexpression of $F L$ NuMA. Hexagon structures were less clear and the vertices were smaller and less well stained. The average interparticle distance for this construct was difficult to measure but seemed to be $\sim 130 \pm 10 \mathrm{~nm}$ (Table 1). Interestingly the cell shown in Fig. $6 \mathrm{~g}$ not only showed a changed nuclear structure but also accumulated NuMA in the cytoplasm. Figures $6 \mathrm{~h}$ and $6 \mathrm{i}$ show a direct comparison at the same magnification of the nuclear lattice and of the cytoplasmic mass formed in a single cell. The cytoplasmic mass shows cables with a smooth side and a fuzzy side (Fig. 6i, see below).

Because some of the features seen with the $\Delta 2005$ transfected cells were reminiscent of apoptotic cells, we used the TUNEL assay. Cells with nuclear aggregates were negative (data not shown) and therefore the observed morphological changes are most probably not due to induction of an apoptotic process.

\section{NuMA Polypeptides with Longer C-Terminal}

Deletions F orm Cytoplasmic Masses with a Hexagonal UItrastructure

The nuclear ultrastructure observed in cells overexpressing FL or certain other NuMA constructs was reminiscent of that of the cytoplasmic masses reported for cells transfected with NuMA- $\Delta$ NLS and then treated with either Taxol or nocodazole. The NuMA- $\Delta$ NLS construct lacks part of the nuclear localization signal due to a deletion covering residues 1985 to 1989 [33].

We have shown previously that NuMA polypeptides truncated before the NLS, i.e., at or before residue 2005, bound to microtubules if the polypeptide was as long as or longer than 1950 amino acid residues (Fig. 7 and [28]). In contrast NuMA polypeptides which terminate at residues 1935 or before do not bind to microtubules (Fig. 6 and [28]). The electron micrographs in Figs. 8a and 8b show that long filaments can be clearly distinguished in the cytoplasmic masses from both the $\Delta 2003$ and the $\Delta 1950$ constructs. These structures were resistant to the detergent extraction. At high magnification such structures had a smooth side and a fuzzy side (Fig. 8b), as already reported for cables of similar diameter in cytoplasmic masses in cells transfected with NuMA- $\Delta$ NLS [33]. Measurements of the diameter of filaments ignoring the fuzzy projections yielded values of $22.4 \pm 2.5 \mathrm{~nm}$ for $\Delta 2005$, $22.3 \pm 1.7 \mathrm{~nm}$ for $\Delta 2003$, and $21.8 \pm 2.2 \mathrm{~nm}$ for $\Delta 1950$. The diameter of the individual rods projecting from the fuzzy side of the $22-\mathrm{nm}$ filaments was $6.0 \pm 0.4 \mathrm{~nm}$. In parallel cultures treated with colcemid for $2 \mathrm{~h}$ and then extracted with detergent, such 22-nm filaments were conspicuously absent and instead structures with a hexagonal arrangement were seen in the cytoplasm (Figs. 8c and 8d). The average center to center distance was $136 \pm$ $11 \mathrm{~nm}$ (Fig. 8d, Table 1). The ultrastructure of cytoplasmic masses found in cells transfected with the NuMA $\Delta 1935$ construct was similar to that found in the cytoplasm of colcemid-treated cells transfected with the $\Delta 1950$ construct (compare Figs. 8e and 8c). However, at higher magnification the hexagonal networks from the $\Delta 1935$ construct are clearly more disorganized, and the vertices are less electron-dense (compare Figs. 8f and 8d). The average center to center distance for the $\Delta 1935$ construct was $128 \pm 10 \mathrm{~nm}$ (Fig. 8f, Table 1). 

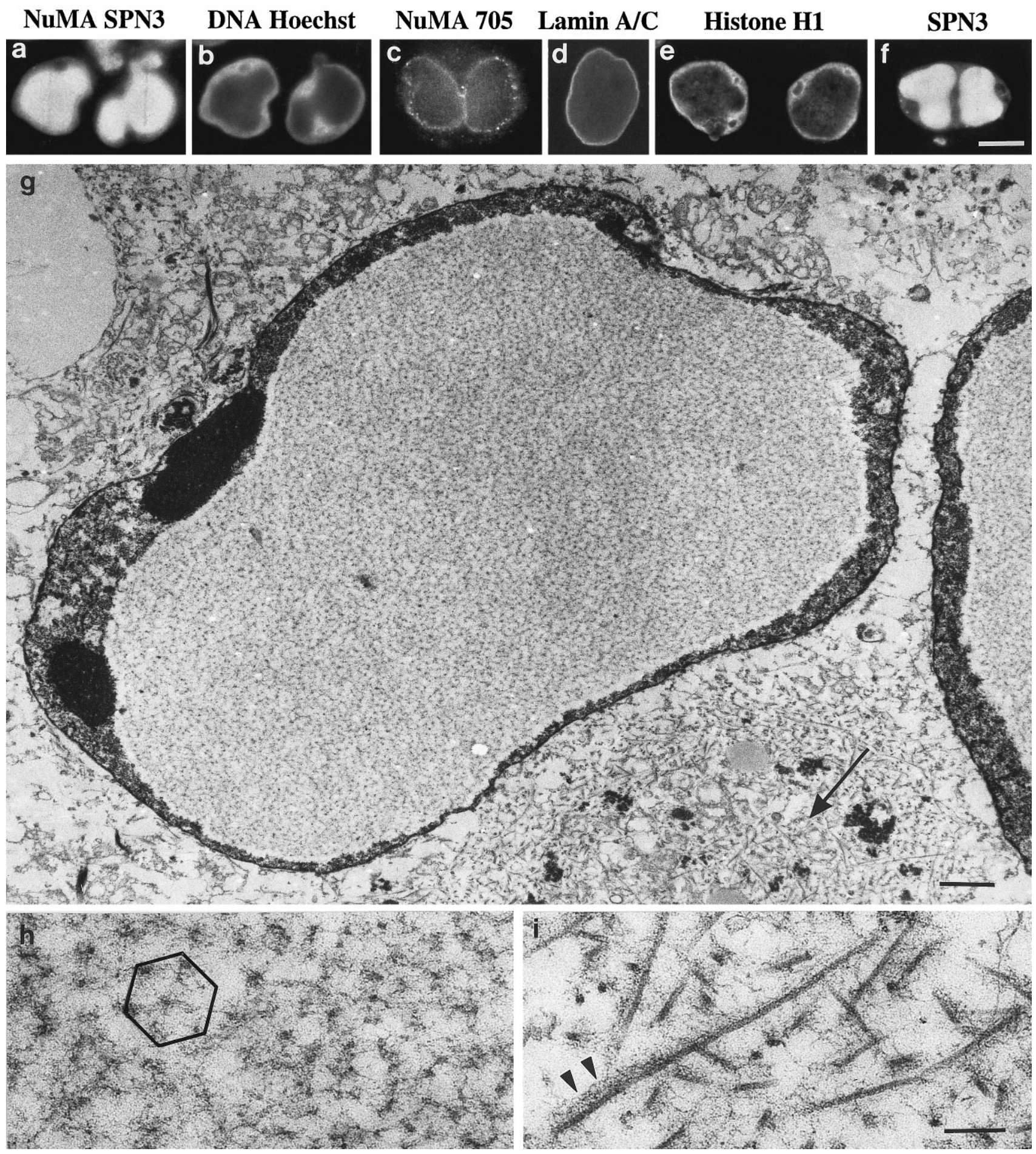

FIG. 6. I mmunofluorescence staining (a-f) and electron microscopy after detergent extraction (g-i) of $\mathrm{HeL}$ a cells $42 \mathrm{~h}$ after transfection with $\Delta 2005$ NuMA cDNA. (a, b) Cells double-stained with NuMA SPN3 antibody (a) and Hoechst dye (b). (c) NuMA 705 staining. (d) Lamin $\mathrm{A} / \mathrm{C}$ staining. (e) Histone $\mathrm{H} 1$ staining. (f) SPN3 staining after detergent extraction. Note that the SPN 3 staining is very strong in the center of the nucleus $(a, f)$, while staining with the 705 antibody which reveals only the endogeneous NuMA surrounds and abuts the SPN 3 staining. DNA, histone $\mathrm{H1}$, and the nucleoli are displaced to the nuclear periphery (b,e). Note in the electron micrograph in (g) the electron-dense material and the nucleoli visible at the nuclear periphery. Hexagons are marked in (h). A higher magnification of the cytoplasmic aggregate indicated by the arrow in (g) is shown in (i). Note cables with the fuzzy side indicated by arrowheads in (i). Bar (a-f) $10 \mu \mathrm{m}$; (g) $1 \mu \mathrm{m}$; ( $\mathrm{h}, \mathrm{i}$ ) $200 \mathrm{~nm}$. 


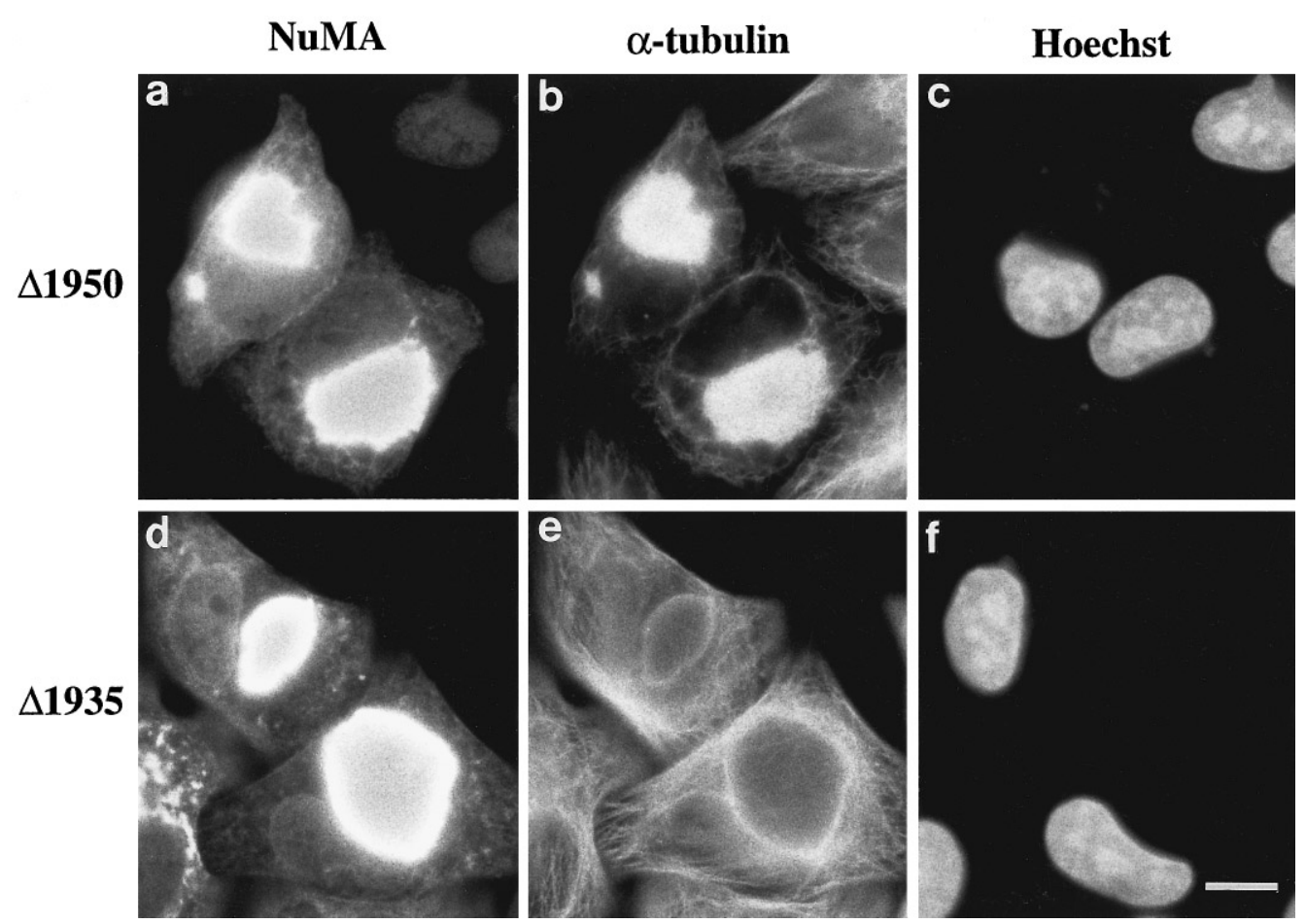

FIG. 7. Immunofluorescence staining of HeLa cells $42 \mathrm{~h}$ after transfection with $\Delta 1950$ (a-c) or $\Delta 1935$ (d-f) NuMA cDNA. (a-f) Triple staining with the NuMA polyclonal antibody 2422 (a, d), $\alpha$-tubulin antibody (b, e), and Hoechst dye (c, f). Note the colocation of the NuMA and $\alpha$-tubulin stains for the $\Delta 1950$ construct $(\mathrm{a}, \mathrm{b})$ and that this is not seen with the $\Delta 1935$ construct (d,e). Bar $10 \mu \mathrm{m}$.

NuMA Polypeptide with a Deletion in the Head and Coiled-Coil Region Also Changes the Nuclear Structure

Finally, we examined the dHCC construct in which amino acids 7-1502 were del eted. This construct lacks almost all of the NuMA head domain and six-sevenths of the coiled-coil domain, but retains the full tail domain (Fig. 1a). The SPN7 antibody recognized this mutant protein in immunoblotting (Fig. 1b) and immunofluorescence (Fig. 9a and 9c), whereas as expected the SPN3 antibody did not. The size of the dHCC polypeptide in immunoblotting analysis was in good agreement with the expected size of $62 \mathrm{kDa}$ (Fig. 1b).

In cells expressing moderate amounts of NuMA $\mathrm{dHCC}$, the overall pattern of NuMA and chromatin in the nucleus remained normal. However, in cells expressing large amounts of the NuMA dHCC polypeptide, the NuMA pattern was clearly changed and multiple NuMA speckles which sometimes had a hole in the center were observed throughout the nucleoplasm (Fig. 9a). These speckles were also visible when the stained specimens were viewed in phase microscopy. Hoechst staining was found in dots centered on the NuMA speckles, suggesting that chromatin localization was disturbed in these cells (Fig. 9b). The dHCC NuMA recombinant clusters resisted extraction with the detergent buffer (Fig. 9c).
When cells transfected with NuMA dHCC were examined by electron microscopy, nuclei with a clearly disturbed ultrastructure were found. No hexagonal organization was detected and instead such cells were characterized by large randomly distributed clusters of chromatin and of proteinaceous material (Fig. 9d).

I mmunogold Labeling of FL Transfected Nuclè

Shows That the Hexagon Structures Contain NuMA

To provide further evidence that the hexagonal structures contain NuMA, FL transfected cells were labeled with the SPN-3 NuMA antibody which recognizes an epitope at the beginning of the coiled-coil region and with second antibodies coupled to nanogold. FL transfected cells were permeabilized with the same buffers used for the electron microscopic studies shown above and were then fixed with either formal dehyde (Fig. 10a) or glutaraldehyde (Fig. 10c). After incubation with both first and second antibodies the nanogold was amplified using a commercial silver development kit. Results are shown in Figs. 10a-10d. In the FL transfected cells hexagonal structures can be identified by their distinctive morphology in both the antibody- treated (Fig. 10a and 10c) and the control samples (Figs. 10b and 10d). Many silverenhanced gold partides are found associated with the hexagonal structures in the FL transfected cells stained 

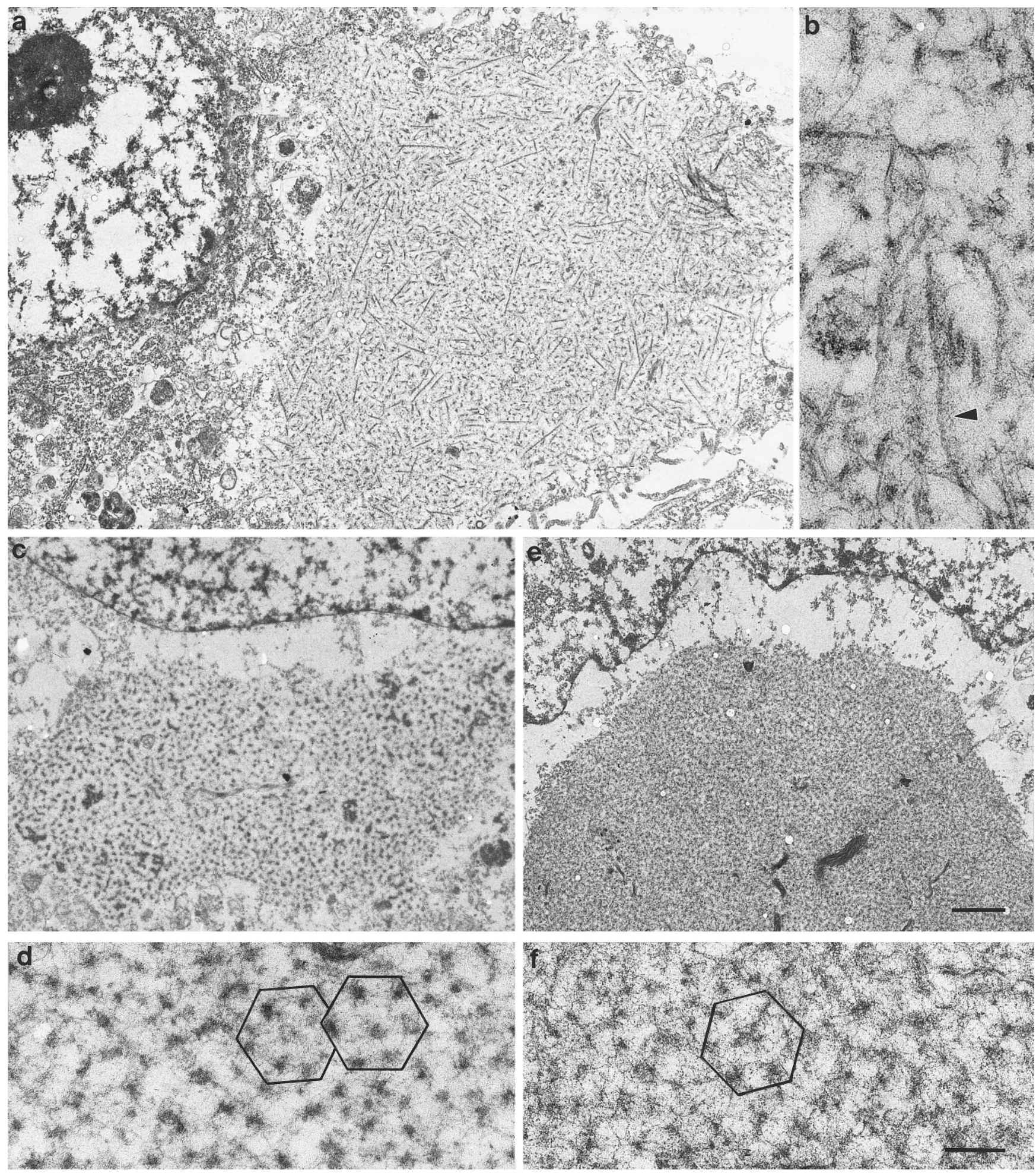

FIG. 8. Electron microscopy of HeLa cells extracted with detergent $42 \mathrm{~h}$ after transfection with $\Delta 2003$ (a), $\Delta 1950$ (b- d), or $\Delta 1935$ (e, f) NuMA cDNA. Cells in (c, d) were colcemid treated. Note the cables in a and b and that these have a smooth side and a fuzzy (arrowhead in b) side. Cables are not present in cells after transfection with $\Delta 1950$ and treatment with colcemid (c, d) or after transfection with the $\Delta 1935$ construct $(e, f)$. Instead the structure resembles that seen in the nucleus with other constructs (Figs. 3-5). At high power the vertices of the hexagons seem less electron-dense than those seen with FL NuMA (d, f). Bar (a, c, e) $1 \mu \mathrm{m}$; (b, d, f) $200 \mathrm{~nm}$. 


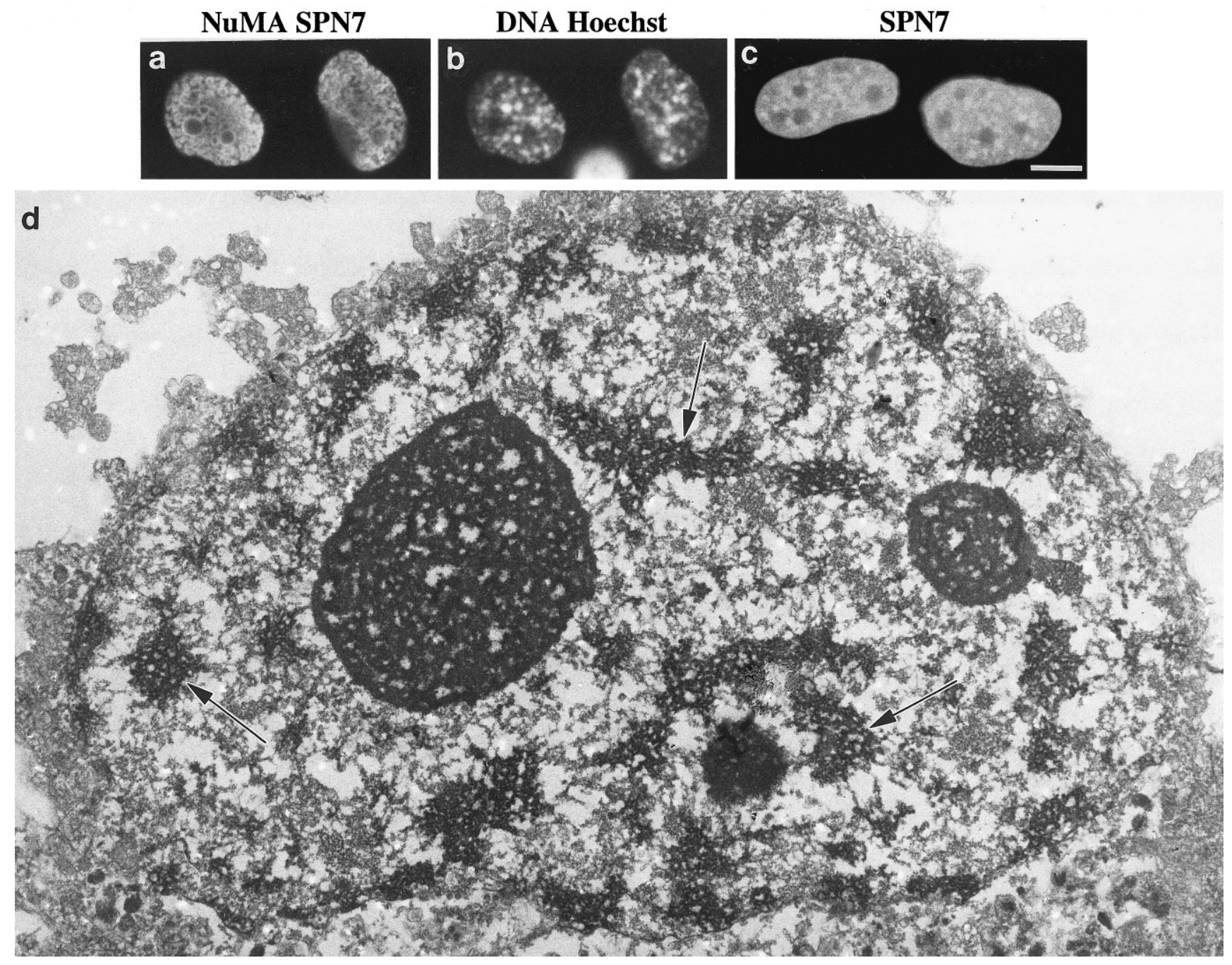

FIG. 9. I Immunofluorescence staining (a-c) and electron microscopy after detergent extraction (d) of $\mathrm{HeL}$ a cells $42 \mathrm{~h}$ after transfection with the dHCC NuMA cDNA in which most of the head and the coiled-coil region have been removed. (a, b) Cells double-stained with NuMA antibody SPN7 (a) and Hoechst dye (b). (c) SPN7 staining after detergent extraction. Note NuMA speckles (a) and that the Hoechst stain (b) shows that DNA is clearly rearranged and coincident with the center of the speckles. The electron micrograph in (d) shows an aberrant arrangement of electron-dense material (arrows) scattered through the nucleoplasm. Bar (a-c) $10 \mu \mathrm{m}$; (d) $1 \mu \mathrm{m}$.

with the NuMA antibodies (Figs. 10a and 10c) but gold particles are only occasionally found associated with hexagonal structures in the FL transfected cells in which the first antibody was omitted (Figs. 10b and 10d). The silverenhanced gold partides have an average size of 14-20 $\mathrm{nm}$ in Fig. 10. The gold-labeled antibodies are found associated with the hexagonal structures throughout the nucleus in both formaldehyde or glutaral deyde-fixed $F L$ transfected cells. Patches of chromatin and the nucleoli are not labeled and very little label was found in the cytoplasm. The gold-labeled SPN3 antibody seems not to decorate the electron-dense vertices of the hexagons but rather to be found between the vertices.

Interphase nuclei of untransfected cells were also examined with NuMA antibodies in the nanogold technique. Representative results in Fig. 10e show clear labeling of the nuclei with SPN-3 antibody while nucleoli, patches of chromatin, and the cytoplasm are not labeled. Almost no labeling is seen if the first antibody is omitted (Fig. 10f). Labeling of the untransfected cells is very much less than that in the transfected cells (compare Figs. 10e and 10c, which are at the same magnification). Again labeling with gold is seen throughout the nucleus and occasional clusters containing two or three gold particles are found. In some areas in the untransfected nuclei gold particles seem separated by a distance of around $200 \mathrm{~nm}$ (see lines drawn on Fig. 2e). However, morphologically distinct electron- dense particles are not present in the micrographs of immunolabeled control cells shown in Fig. $2 e$. This may suggest either that NuMA has a different arrangement in the untransfected cell or that its ar- 


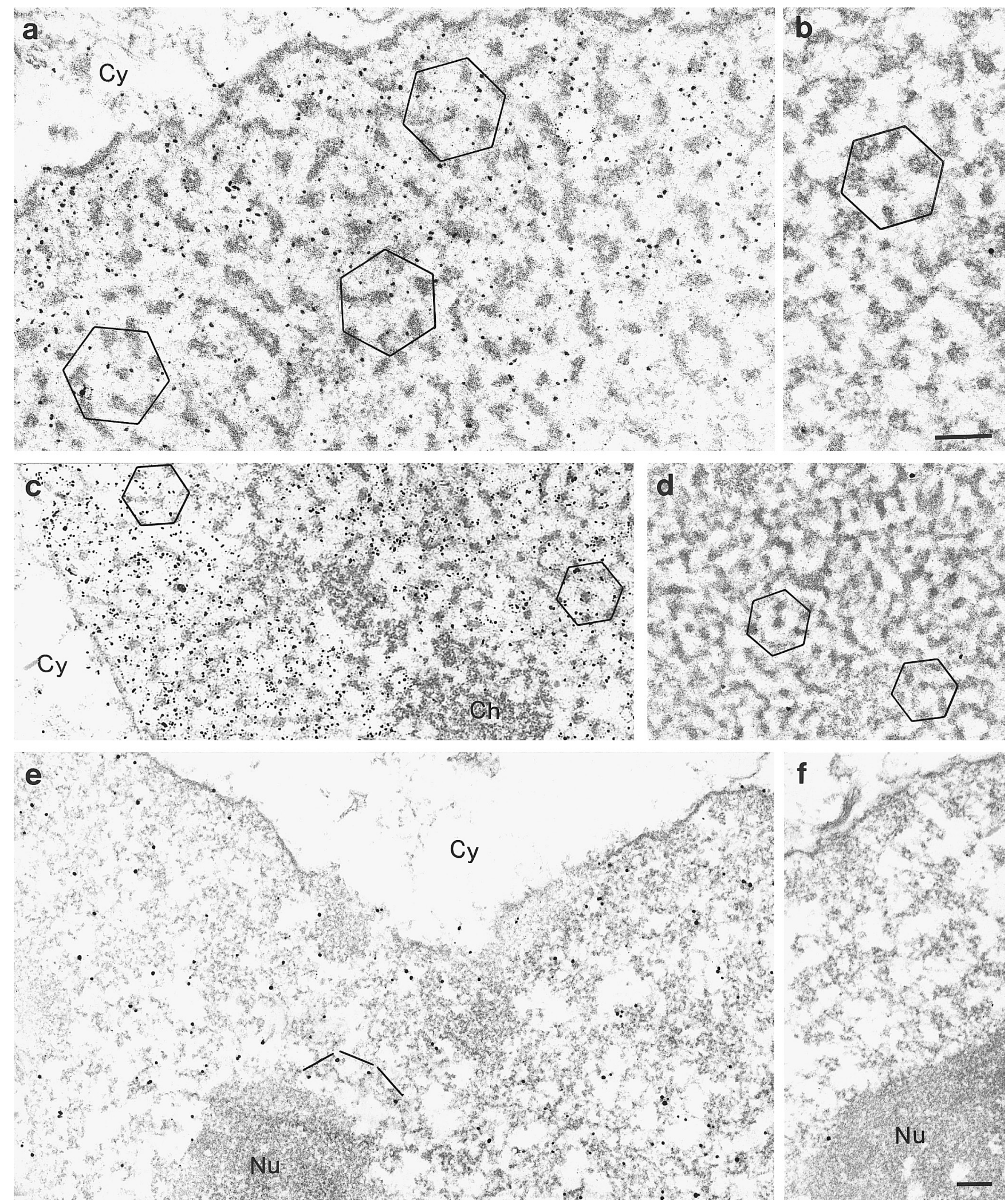

FIG. 10. I mmunogold labeling with the NuMA SPN3 antibody. (a) FL transfected cells fixed with formaldehyde and label ed with NuMA antibody. (b) Control for (a) without NuMA antibody. (c) FL transfected cell fixed with glutaraldehyde and labeled with NuMA antibody. (d) Control for (c) without NuMA antibody. (e) Untransfected cell fixed with formaldehyde and labeled with NuMA antibody. (f) Control for (e) without NuMA antibody. Note that the transfected cells show much higher labeling for NuMA than do the untransfected cells and that in (a) and (c) the label is associated with the hexagonal structures. Chromatin ( $\mathrm{Ch})$, the nucleoli (Nu), and the cytoplasm (Cy) are unlabeled. In the untransfected cell in (f) the lines drawn on the figure are $200 \mathrm{~nm}$ (see text). Bar (a, b) $200 \mathrm{~nm}$; (c-f) $200 \mathrm{~nm}$. 
rangement, if it originally resembled that seen in the transfected cells, might be perturbed by the detergent extraction and subsequent processing for immunoelectron microscopy. Further experiments will determine which of these possibilities is correct.

\section{DISCUSSION}

The main finding in this paper is that interphase cells overexpressing FL NuMA or certain mutant NuMA polypeptides display a three-dimensional lattice within the nuclei which is resistant to detergent extraction and which can be characterized by electron microscopy. The lattice is built from electron-dense particles with quasi-hexagonal packing which in favorable sections appear to be separated by thin rod-like elements. This lattice is intriguing for two reasons. First, its assembly is not based on simple linear filaments, but rather on a space-filling geodesic dome-type assembly. Second, this organization may provide a prototype helpful in understanding nuclear structure in the normal cell.

\section{Evidence That the Nuclear Lattice Seen in \\ Transfected Cells Is Built from NuMA}

There are several lines of evidence indicating that the nuclear lattices are built from NuMA and that the thin 6-nm rods joining the particles are associated with the coiled-coil domains and the electron-dense particles are associated with the NuMA globular domains. First, nuclei of the type shown in Figs. 4-6 which appear filled with the nuclear lattice have been found only in HeLa cells transfected with NuMA cDNAs which highly overexpress NuMA. Thus far they have not been reported in normal or in mock transfected cells, either in this study or in other studies of nuclear structure after detergent extraction $[4,7,25]$. Second, the center to center particle distance measured in electron micrographs varies depending on which NuMA construct was used (Table 1 and next section), suggesting that the thin 6-nm rods joining the particles may be associated with the coiled-coil domains. Third, the average diameter of the particles at the hexagon vertices varies depending on which construct was used, suggesting that the electron-dense particles may be associated with the NuMA globular domains (Table 1 and next section). Fourth, the nuclear aggregates share similarities in ultrastructure with a subset of cytoplasmic N uMA aggregates in BHK-21 cells shown by Saredi et al. [33] to contain only NuMA- $\Delta$ NLS polypeptides (see below for further discussion). Finally, immunoelectron microscopy (Fig. 10) with antibodies to NuMA results in lattices that are strongly and specifically labeled in the immunogold technique.
Lattice Parameters Vary Depending on the NuMA Construct

The measured center to center particle distances and the measured particle diameters for the different NuMA constructs are summarized in Table 1 . The measured center to center particle distances show a qualitative agreement with the distances calculated from the length of the coiled-coils, assuming a value of $0.1485 \mathrm{~nm}$ per coiled-coil residue. Thus CCXL, which has an $80 \%$ addition in the putative coiled-coil region, has the largest center to center particle spacing (236 $\mathrm{nm}$ ). This is longer than the FL center to center particle spacing (166 nm). The dCC 1 and dCC2 constructs, which have deletions of 40 and $60 \%$ of the coiled-coil regions, respectively, show shortened center to center spacings of $135 \pm 20 \mathrm{~nm}$ and $102 \pm 13 \mathrm{~nm}$, respectively. These distances are considerably shorter than the spacing seen in nuclei overexpressing FL NuMA.

The measured particle diameters also show a qualitative agreement with the diameters that would be expected if the length of the tail regions of the different constructs are taken into account. Thus the $F L$ and the CCXL constructs which retain the full tail domain have the largest particle diameter, while the $\Delta 2005, \Delta 1950$, and $\Delta 1935$ constructs show smaller particle diameters (Table 1). However, the dCC1 and dCC2 constructs which retain the full tail domain also show decreased particle diameters. One possible explanation may be that proteins that bind to the C-terminal region of the NuMA molecule may contribute to the particle diameter. Because the dCC1 and dCC2 constructs have a closer packing than the $\mathrm{F} L$ and CCXL constructs, more particles are present and there might not be enough of the putative C-terminal binding proteins to saturate the vertices of the dCC1 and dCC2 constructs.

All the constructs show measured lengths which are considerably shorter (54-80\%) than the lengths that would be expected from the calculated coiled-coil lengths plus the particle diameter (Table 1 ). The FL interparticle distance, which is measured at $166 \pm 14$ $\mathrm{nm}$, is also shorter than the length of $207 \pm 20 \mathrm{~nm}$ measured for the NuMA rod region from rotary shadowed images of the FL NuMA molecule expressed in Escherichia coli [32].

NuMA forms a three-dimensional quasi-hexagonal array in transfected cells. The hexagonal arrays seen in cells transfected with the FL construct, or with certain other constructs discussed above, are not absolutely regular. This "irregularity" in the lattice could either be an inherent property of thestructure or be caused by the fact that the cells are extracted with detergent prior to fixation for electron microscopy. Alternatively the irregularity could be caused by the angle at which the cell is cut and the fact that sectioning of the cells gives only a two-dimensional view of the structure. 


\section{Assembly of NuMA into Lattices}

It is also interesting to compare NuMA with the yeast spindle body proteins Spc110p [40] coded for by the NUF 1 gene [41] and Spc42p [42]. Both proteins have a central coiled-coil rod flanked by globular domains, although both coiled-coil domains are much shorter than that of NuMA. Interestingly a discrepancy was also seen for the Spc110p protein between the length of the rod domain as observed on rotary shadowed micrographs (78 $\pm 9 \mathrm{~nm})$ and the edge to edge spacing between particles (60 nm) [43]. Spc110p plays an essential role in mitotic spindle formation $[43,44]$. A Spcl10p-related protein has been identified in vertebrates. It is associated with the centrosome and is involved in microtubule nucleation [45]. A Spc42p crystal may form the core of the native spindle pole body and the protein has an essential function during spindle pole body duplication [42].

The assembly properties of N UMA are reminiscent of those of Spc110p and Spc42p [46]. Indeed the hexagonal arrangements of N uMA demonstrated here resemble those seen when Spc110p was overexpressed [43]; however, the NuMA networks are much more extensive. Kilmartin and Goh [43] propose that antiparallel Spc110p molecules interact through their C-terminal domains clustered in the electron-dense particles to produce a three-dimensional network. The fact that we observe a dotted rather than a homogeneous NuMA immunofluorescent pattern when using the NuMA 705 and c-myc antibodies which recognize epitopes close to the NuMA C-terminus would also suggest that the C-terminal domains of the NuMA molecules might be found in the electron-dense particles. Current data do not, however, exclude other models for how NuMA assembles into the lattices we observe in transfected cells.

Rearrangement of Normal Nuclear Constituents in

Cells Transfected with Certain NuMA Constructs

We stress the parallels seen when cells overexpressing the same NuMA constructs are examined by immunofluorescence microscopy using NuMA antibodies and by electron microscopy. Cells overexpressing $F L$ NuMA (Fig. 4) or one of the NuMA constructs with additions or deletions in the coiled-coil (cf. CCXL and dCC2 in Fig. 5) show homogenous staining of the nucleoplasm with the NuMA SPN3 antibody. Nuclei filled with an ordered lattice can be found in electron micrographs of cells transfected with the same constructs. In contrast light microscopy of cells overexpressing the C-terminally truncated constructs $\Delta 2030$ and $\Delta 2005$, which retain the NLS, shows that recombinant NuMA polypeptides (as assayed by SPN3 staining) are concentrated in one or more large aggregates which almost fill the nucleus, while the DNA, histone $\mathrm{H} 1$, and endogenous NuMA polypeptides (as assayed by 705 antibody staining) together with the nucleoli are displaced to a very narrow rim on the inner side of the nuclear membrane. Electron microscopy provides proof of the rearrangement of the nuclear constituents. It shows less densely stained but apparently structured material in the center, with the nucleoli and more electron-dense material displaced to the outer rim of the nucleus (Fig. 6g). Cells overexpressing the dHCC construct in which most of the NuMA head and coiledcoil domains are deleted also show a changed nuclear structure. In immunofluorescence microscopy the dHCC construct is found in multiple speckles and the DNA distribution is altered (Figs. 9a and 9b) while in electron microscopy apparently aberrant arrangements of electron-dense material scattered throughout the nucleoplasm are detected (Fig. 9d).

\section{NuMA Cytoplasmic Aggregates and Interaction of NuMA with Microtubules}

Cytoplasmic aggregates of NuMA can be detected under two conditions. First, such aggregates can be found in $5-10 \%$ of FL transfected cells by immunofluorescence microscopy. Second, if NuMA constructs lacking the NLS are used, NuMA cannot enter the nucleus. Instead NuMA accumulates in the cytoplasm where it forms a single large aggregate which usually surrounds, or is close to, the centrosome [33, 28]. Electron microscopy of cytoplasmic aggregates in BHK 21 cells transfected with the NuMA- $\Delta$ NLS construct showed cables with a diameter of $\sim 23 \mathrm{~nm}$ with fuzzy and smooth sides, as well as short filaments with a diameter of $\sim 5 \mathrm{~nm}$ [33]. A similar ultrastructure was found for cytoplasmic aggregates in cells transfected with the NuMA $\Delta 1950$ construct (Fig. 8b). This terminally truncated construct also lacks the NLS. Cables were not observed either in cells transfected with NuMA- $\Delta$ NLS and then treated with nocodazole or Taxol to destroy microtubules [33] or in cells transfected with NuMA $\Delta 1950$ and then treated with colcemid to destroy microtubules (Figs. 8c and 8d). Instead arrays of electron-dense foci connected by 5 to $6-\mathrm{nm}$ filaments were seen with an ultrastructure which shares features with that reported here for the NuMA nuclear lattice. Saredi et al. [33, 34] have isolated the cytoplasmic aggregates by flow cytometry and shown that after isolation these aggregates contain only NuMA- $\Delta$ NLS polypeptides, and these authors have also reported similar aggregates in mitotic extracts.

Of particular interest in the current study is that cables were not found in the cytoplasmic aggregates in cells transfected with the $\Delta 1935$ construct, even if the cells were not pretreated with colcemid (Figs. 8e and 8f). Our data are consistent with the idea that the 22-nm cables are microtubules to which NuMA binds 
as 5- to 6-nm- diameter filaments which extend outward from the fuzzy side of the microtubule. Both the diameter of the cables, and the sensitivity to nocodazole and colcemid would support this interpretation. However, we cannot exclude other interpretations of cable structures and we note the possibility raised by Saredi et al. [33] that in the cables the microtubules might open laterally into curved sheets. In addition NuMA is known to associate with microtubules of the mitotic spindle in vivo (see Refs. cited in Introduction) and to bind to Taxol-stabilized microtubules in vitro $[21,47]$.

Light microscopic studies suggested that cytoplasmic NuMA aggregates formed by tail truncated constructs equal to or longer than $\Delta 1950$ stain positively for tubulin, while aggregates formed by $\Delta 1935$ or shorter constructs do not (Table 1 and [28]). The light microscopic data in Fig. 7 and the electron microscopic data in Fig. 8 show that the region between NuMA residues 1935 and 1950 is directly or indirectly required for microtubule binding. The sequence ${ }_{1930} \mathrm{CP}$ PHLKTCYPLESRPSLSLGT 1950 is highly conserved in human and Xenopus NuMA. The first 17 residues are identical except for the replacement of a $\mathrm{C}$ at position 1937 in the human sequence by an $S$ and of the $L$ at position 1946 by an I in the Xenopus sequence. The sequence does not show similarity to microtubular binding sites thus far identified in other microtubulerassociated proteins such as MAP2 and tau [48]. In addition the realization that NuMA, when overexpressed, can bind to cytoplasmic microtubules of interphase cells may provide an explanation for the structures identified in the light microscope as filaments or rods in a minority of the cells overexpressing $\mathrm{FL}$ or certain C-terminally truncated constructs [28].

A further intriguing question is: why do the cytoplasmic NuMA aggregates associate with the centrosome? Excess NuMA polypeptides that are unable to enter the nucleus either because the nuclear binding sites for NuMA are saturated (e.g., for cells transfected with the FL NuMA) or because the NuMA construct lacks a nuclear localization signal (e.g., $\Delta 2003, \Delta 1950, \Delta 1935$ ) are retained in the centrosomal region. This may be not only because NuMA binds to microtubules but could also involve interactions of the long coiled-coil region present in the NuMA molecule with other constituents in the centrosomal region. We note that certain other proteins which localize to the centrosome in interphase, i.e., ninein and pericentrin, also have long coiled-coil regions [49, 50].

Finally, one should consider whether lattices of the type seen in the nucleus under conditions in which NuMA is overexpressed might also be important at the mitotic spindle poles. Merdes et al. [20] suggested that NuMA interacts both with microtubules and with microtubule motors such as cytoplasmic dynein and dyn- actin to tether spindle microtubules to the pole. One way in which this might be done would be if NuMA would self-assemble into lattices of limited extent with a focus at each spindle pole.

\section{Arrangement and Possible Function of NuMA in the Normal Interphase Nucleus}

Our results showing that an ordered NuMA lattice is assembled in the nucleus of transfected cells and its similarity to that seen in the cytoplasm with a N uMA$\Delta \mathrm{NLS}$ construct suggest that interacting proteins may not be essential to build this structure.

Truncation of the last 85 amino acid residues of the tail domain in $\Delta 2030$, or of the last 110 residues of the tail domain in $\Delta 2005$, results in the segregation of histones H1, DNA, and nucleoli to the inner rim of the nuclear membrane, while the mutant NuMA molecules aggregate in the center of the nuclei of transfected cells. This suggests that the NuMA network may form or be part of an essential structural support to which other molecules such as chromatin or histones may bind and that NuMA molecules that lack 85 or more residues of the tail cannot perform this function. In accordance with this result, NuMA has been previously shown to bind specifically to matrix attachment regions of DNA in vitro [29] and to interact with histone $\mathrm{H} 1.2$ in the two-hybrid system [54]. Induction of apoptosis results in cleavage of NuMA in the tail domain and in dissociation of NuMA from the chromatin [5153]. Despite the drastic reorganization of nuclear components induced with the NuMA constructs $\Delta 2005$ (Fig. 6) or dHCC (Fig. 9), the nuclear lamina and the nucleolus seemed normal. This suggests that the different compartments of the nucleus, i.e., the lamina at the periphery, the internal nuclear matrix, and the nucleolus, may act independently to organize the nuclear structure.

Interphase nuclei competent to replicate their DNA and to import nuclear substrates have been recently assembled from human sperm DNA in frog cell extracts depleted of NuMA proteins [23]. Our results showing that the lamina and the internal matrix organize independently are in accordance with this competence to form functional nuclear pores in the absence of NuMA. While most normal cell and tissue types that have been tested have NuMA [21, 22], a few cell types with nonspherical nuclei such as granulocytes and certain smooth and skeletal muscle cell types have been reported as lacking NuMA [23]. Further experiments will show whether NuMA is indeed not present in such cells or whether such cells might be characterized by the presence of alternatively spliced NuMA forms [22] not detected by the antibodies used in [23]. Alternatively, perhaps the lack of NuMA might be responsible for the aberrant nuclear shape [23]. 
How is NuMA arranged in the normal interphase nucleus? In micrographs of mock transfected nuclei extracted with detergent (e.g., Fig. 3), occasional electron-dense foci can be visualized, but no extensive regular quasi-hexagonal lattices of the type detected in cells transfected with FL NuMA or with NuMA constructs (Figs. 4- 6 and 8) have been detected. The micrographs of untransfected cells show a relatively empty cytoplasm with few of the straight or branched filaments noted by other researchers using different detergent protocols. Interphase nuclei are clearly specifically labeled when NuMA antibodies are used in the nanogold technique (Fig. 10). In some micrographs gold particles with a spacing of around $200 \mathrm{~nm}$ can be found, and clusters of two or sometimes three gold particles are found. Untransfected cells have some 40fold less NuMA than do the FL NuMA transfected cells ([28] and Fig. 1b). This has two consequences important for attempts to understand the distribution of NuMA in the normal interphase nucleus. First, any higher order structures formed by NuMA in the normal interphase nucleus may be more sensitive to detergent extraction and to the procedures used for electron microscopy than are the lattices seen in the transfected cells which fill the nucleus. Second, if endogenous NuMA would polymerize into lattices similar to those detected in Figs. 4-6, such lattices would not extend throughout the normal nucleus but would be spatially restricted. One possiblity is that endogenous NuMA could self-assemble at least in part to form multiple discrete "minilattices," each of which contains multiple NuMA molecules arranged in a similar ultrastructure.

Our findings on cells transfected with different NuMA constructs raise several questions relevant to the study of the nuclear matrix of the normal nucleus. First, there is the idea that the nuclear matrix could be based at least in part on the formation of discrete three- dimensional lattices or cages composed of electron-dense foci joined by thin rods rather than on the long linear or branched filaments traversing the nucleus reported in previous studies of detergent extracted nuclei. Second, coiled-coil proteins such as NuMA might form the building block(s) of such a matrix because of their ability to self- assemble locally into such lattices. Third, such lattices, if locally re stricted might serve to compartmentalize the nucleus and provide frameworks or cages on or in which to hang other molecules or on which functional activities of the nucleus might take place.

We thank Heinz-J ürgen Dehne for help with the electron microscopy. We are especially grateful to Christina Grund, German Cancer Research Center, Heidelberg who introduced us to the nanogold labeling technique.

\section{REFERENCES}

1. Berezney, R., and Coffey, D. S. (1977). Nuclear matrix: I solation and characterization of a framework structure from rat liver nuclei. J . Cell Biol. 73, 616-637.

2. Belgrader, P. A., Siegel, J ., and Berezney, R. (1991). A comprehensive study on the isolation and characterization of the HeLa S3 nuclear matrix. J . Cell Sci. 98, 281-291.

3. Mirkovitch, J., Mirrault M.-E., and Laemmli, U. K. (1984). Organization of the higher-order chromatin loop: Specific DNA attachment sites on nuclear scaffold. Cell 39, 223-232.

4. J ackson, D. A., and Cook, P. R. (1988). Visualization of a filamentous cytoskeleton with a $23 \mathrm{~nm}$ axial repeat. EMBO J. 7, 3667-3677.

5. Stuurman, N. A., Meijne, M. L., van der Pol, A. J ., de J ong, van Driel, R., and van Renswoude, J. (1990). The nuclear matrix from cells of different origin: Evidence for a common set of matrix proteins. J . Biol. Chem. 265, 5460-5465.

6. Fey, E. G., Krochmalnic, G., and Penman, S. (1986). The nonchromatin substructures of the nucleus: The ribonucleoprotein (RNP)-containing and RNP-depleted matrices analyzed by sequential fractionation and resinless electron microscopy. J . Cell Biol. 102, 1654-1665.

7. He, D., Nickerson, J . A., and Penman, S. (1990). Core filaments of the nuclear matrix. J . Cell Biol. 110, 569-580.

8. Cook, P. R. (1988). The nucleoskeleton: Artefact, passive framework or active site? J . Cell Sci. 90, 1-6.

9. Berezney, R., Mortillaro, J ., Ma, H., Wei, X., and Samarabandu J . (1995). The nuclear matrix: A structural milieu for genomic function. Int. Rev. Cytol. 162A, 1-65.

10. van Driel, R., Wansink, D. G., van Steensel, B., Grande, M. A., Schul, W., and de J ong, L. (1995). Nuclear domains and the nuclear matrix. Int. Rev. Cytol. 162A, 151-189.

11. Mattern, K. A., Humbel, B. M., Muijsers, A. O., de J ong, L., and van Driel, R. (1996). hnRNP proteins and B23 are the major proteins of the internal matrix of HeLa S3 Cells. J . Cell Biochem. 62, 275-289.

12. Hozak, P., Sasseville, A. M.-J ., Raymond, Y., and Cook, P. R. (1995). Lamin proteins form an internal nucleoskeleton as well as a peripheral lamina in human cells. J . Cell Sci. 108, 635644.

13. Lydersen, B., and Pettijohn, D. (1980). Human-specific nuclear protein that associates with the polar region of the mitotic apparatus: Distribution in a human/hamster hybrid cell. Cell 22, 489- 499.

14. Kallajoki, M., Weber, K., and Osborn, M. (1991). A 210 kDa nuclear matrix protein is a functional part of the mitotic spindle: A microinjection study using SPN monoclonal antibodies. EMBO J . 10, 3351-3362.

15. Compton, D. A., Szilak, I., and Cleveland, D. W. (1992). Primary structure of NuMA, an intranuclear protein that defines a novel pathway for the segregation of proteins at mitosis. J . Cell Biol. 116, 1395-1408.

16. Kallajoki, M., Harborth, J., Weber, K., and Osborn, M. (1993). Microinjection of a monoclonal antibody against SPN antigen, now identified by peptide sequences as the NuMA protein, induces micronuclei in PtK 2 cells. J .Cell. Sci. 104, 139-150.

17. Yang, C. H., and Snyder, M. (1992). The nuclear-mitotic protein is important in the establishment and maintenance of the bipolar mitotic spindle apparatus. Mol. Biol. Cell 3, 1259-1267.

18. Gaglio, T., Saredi, A., and Compton, D. A. (1995). NuMA is required for the organization of microtubules into aster-like mitotic arrays. J . Cell. Biol. 131, 693-708. 
19. Compton, D. A., and Luo, C. (1995). Mutations in the predicted p34 ${ }^{\mathrm{cdc}}$ phosphorylation sites in NuMA impair the assembly of the mitotic spindle and block mitosis. J . Cell Sci. 108, 621-633.

20. Merdes, A., Ramyar, K., Vechio, J. D., and Cleveland D. W. (1996). A complex of NuMA and cytoplasmic dynein is essential for mitotic spindle assembly. Cell 87, 447- 458.

21. Kallajoki, M., Weber, K., and Osborn, M. (1992). Ability to organize microtubulues in taxol-treated mitotic PtK 2 cells goes with the SPN antigen and not with the centrosome. J . Cell Sci. 102, 91-102.

22. Tang, K. T., Tang, C. C., Chen, Y.-L., and Wu, C.-W. (1993). Nuclear proteins of the bovine esophageal epithelium. II. The NuMA gene gives rise to multiple mRNAs and gene products reactive with monoclonal antibody W1. J . Cell Sci. 104, 249260.

23. Merdes, A., and Cleveland, D. W. (1998). The role of NuMA in the interphase nucleus. J . Cell Sci. 111, 71-79.

24. Zeng, C., He, D., and Brinkley, B. R. (1994). Localization of NuMA protein isoforms in the nuclear matrix of mammalian cells. Cell Motil. Cytoskeleton 29, 167-176.

25. Nickerson, J. A., Blencowe, B. J ., and Penman, S. (1995). The architectural organization of nuclear metabolism. Int. Rev. Cytol. 162A, 67-123.

26. Hozak, P. (1996). The nucleoskeleton and attached activites. Exp. Cell Res. 229, 267-271.

27. Compton, D. A., and Cleveland, D. W. (1993). NuMA is required for the proper completion of mitosis. J . Cell Biol. 116, 13951408.

28. Gueth-Hallonet, C., Weber, K., and Osborn, M. (1996). NuMA: A bipartite nuclear location signal and other functional properties of the tail domain. Exp. Cell Res. 225, 207-218.

29. Ludérus, M. E. E., den Blaauwen, J . L., de Smidt, O. J. B., Compton, D. A., and van Driel, R. (1994). Binding of matrix attachment regions to lamin polymers involves singlestranded regions and the minor groove. Mol. Cell. Biol. 14, 6297- 6305.

30. Yang, C. H., Lambie, E. J ., and Snyder, M. (1992). NuMA: An unusually long coiled-coil related protein in the mammalian nucleus. J . Cell Biol. 116, 1303-1317.

31. Parry, D. A. D. (1994). NuMA/centrophilin: Sequence analysis of the coiled-coil rod domain. Biophysics 67, 1203-1206.

32. Harborth, J., Weber, K., and Osborn, M. (1995). Epitope mapping and direct visualization of the parallel, in-register arrangement of the double stranded coiled-coil in the NuMA protein. EMBO J . 14, 2447-2460.

33. Saredi, A., Howard, L., and Compton, D. A. (1996). NuMA assembles into an extensive filamentous structure when expressed in the cell cytoplasm. J . Cell Sci. 109, 619-630.

34. Saredi, A., Howard, L., and Compton, D. (1997). Phosphorylation regulates the assembly of NuMA in a mammalian mitotic extract. J . Cell Sci. 110, 1287-1297.

35. Chen, C., and Okayama, H. (1987). High efficiency transformation of mammalian cells by plasmid DNA. Mol. Cell Biol. 7, 2745-2752.

36. Osborn, M., and Weber, K. (1977). The display of microtubules in transformed cells. Cell 12, 561-571.

37. Wehland, J ., and Weber, K. (1987). Turnover of the carboxyterminal tyrosine of $\alpha$-tubulin and means of reaching elevated levels of detyrosination in living cells. J . Cell Sci. 3, 1259-1267.

38. Röber, R.-A., Gieseler, R. K. H., Peters, J . H., Weber, K., and Osborn, M. (1990). Induction of nuclear lamins A/C in macro- phages in in vitro cultures of rat bone marrow precursor cells and human blood monocytes, and in macrophages elicited in vivo by thioglycollate stimulation. Exp. Cell Res. 190, 185-194.

39. Rose, O., Grund, C., Reinhardt, S., Starzinski-Powitz, A., and Franke, W. W. (1995). Contactus adherens, a special type of plaque-bearing adhering junction containing M-cadherin, in the granule cell layer of the cerebellar glomerulus. Proc. Natl. Acad. Sci. USA 92, 6022- 6026.

40. Kilmartin, J. V., Dyos, S. L., Kershaw, D., and Finch, J. T. (1993). A spacer protein in the Saccharomyces cerevisiae spindle pole body whose transcript is cell cycle-regulated. J . Cell Biol. 123, 1175-1184.

41. Mirzayan, C., Copeland, C. S., and Snyder, M. (1992). The NUF 1 gene encodes an essential coiled-coil related protein that is a potential component of the yeast nucleoskeleton. J . Cell Biol. 116, 1319-1332.

42. Donaldson, A. D., and Kilmartin, J . V. (1996). Spc42p: A phosphorylated component of the $S$. cerevisiae spindle pole body (SPB) with an essential function during SPB duplication. J . Cell Biol. 132, 887-901.

43. Kilmartin, J. V., and Goh, P.-Y. (1996). Spc110p: Assembly properties and role in the connection of nuclear microtubules to the yeast spindle pole body. EMBO J . 15, 4592- 4602.

44. Sundberg, H. A., Goetsch, L., Byers, B., and Davis, T. N. (1996). Role of calmodulin and Spc110p interaction in the proper assembly of spindle pole body components. J . Cell Biol. 133, 111-124.

45. Tassin, A. M., Celati, C., Paintrand, M., and Bornens, M. (1997). Identification of an Spc110p-related protein in vertebrates. J . Cell Sci. 110, 2533-2545.

46. Bullitt, E., Rout, M. P., Kilmartin, J . V., and Akey, C. W. (1997). The yeast spindle pole body is assembled around a central crystal of Spc42p. Cell 89, 1077-1086.

47. Maekawa, T., and Kuriyama, R. (1993). Primary structure and microtubule interacting domain of the SP-H antigen: A mitotic MAP located at the spindle pole and characterized as a homologous protein to NuMA. J . Cell Sci. 105, 589-600.

48. Lewis, S. A., Wang, D. H., and Cowan, N. J . (1988). Microtubule-associated protein MAP2 shares a microtubule binding motif with tau protein. Science 242, 936-939.

49. Bouckson-Castaing, V., Moudjou, M., Ferguson, D. J . P., Mucklow, S., Belkaid, Y., Milon, G., and Crocker, P. R. (1996). Molecular characterisation of ninein, a new coiled-coil protein of the centrosome. J . Cell Sci. 109, 179-190.

50. Doxsey, S. J ., Stein, P., E vans, L., Calarco, P. D., and Kirschner, M. (1994). Pericentrin, a highly conserved centrosome protein involved in microtubule organization. Cell 76, 639- 650.

51. Hsu, H-L., and Yeh, N-H. (1996). Dynamic changes of NuMA during the cell cycle and possible appearance of a truncated form of NuMA during apoptosis. J . Cell Sci. 109, 277-288.

52. Weaver, V. M., Carson, C. E., Walker, P. R., Chaly, N., Lach, B., Raymond, Y., Brown, D. L., and Sikorska, M. (1996). Degradation of nuclear matrix and DNA cleavage in apoptotic thymocytes. J . Cell Sci. 109, 45-56.

53. Gueth-Hallonet, C., Weber, K., and Osborn, M. (1997). Cleavage of the nuclear matrix protein NuMA during apoptosis. Exp. Cell Res. 233, 21-24.

54. McPherson, S. M., Matova, N., and Snyder, M. (1994). ASCB Meeting, Abstract 1986. 